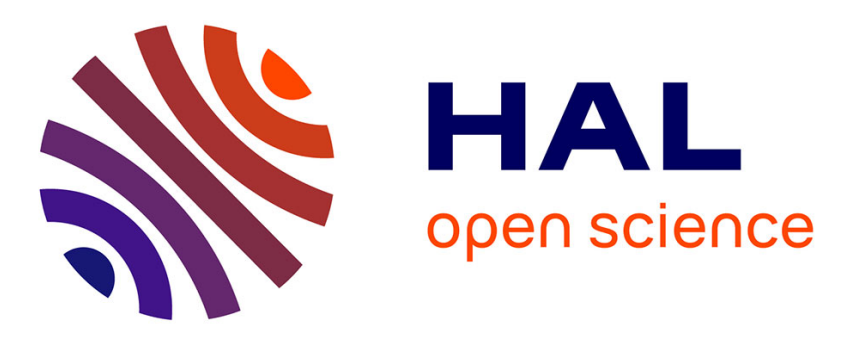

\title{
Model and methods to assess hepatic function from indocyanine green fluorescence dynamical measurements of liver tissue
}

Chloe Audebert, Irene Vignon-Clementel

\section{- To cite this version:}

Chloe Audebert, Irene Vignon-Clementel. Model and methods to assess hepatic function from indocyanine green fluorescence dynamical measurements of liver tissue. European Journal of Pharmaceutical Sciences, 2018, 10.1016/j.ejps.2018.01.008 . hal-01696064

\section{HAL Id: hal-01696064 https://hal.science/hal-01696064}

Submitted on 30 Jan 2018

HAL is a multi-disciplinary open access archive for the deposit and dissemination of scientific research documents, whether they are published or not. The documents may come from teaching and research institutions in France or abroad, or from public or private research centers.
L'archive ouverte pluridisciplinaire $\mathbf{H A L}$, est destinée au dépôt et à la diffusion de documents scientifiques de niveau recherche, publiés ou non, émanant des établissements d'enseignement et de recherche français ou étrangers, des laboratoires publics ou privés. 


\title{
Model and methods to assess hepatic function from indocyanine green fluorescence dynamical measurements of liver tissue*
}

\author{
Chloe Audebert ${ }^{\mathrm{a}, \mathrm{b}}$, Irene E. Vignon-Clementel ${ }^{\mathrm{a}, \mathrm{b}, *}$ \\ ${ }^{a}$ Inria centre de recherche de Paris, 2 rue Simone Iff, 75012 Paris, France \\ ${ }^{b}$ Sorbonne Universités, UPMC, Laboratoire Jacques-Louis Lions, 4 place Jussieu, 75005 Paris, France
}

\begin{abstract}
The indocyanine green (ICG) clearance, presented as plasma disappearance rate is, presently, a reliable method to estimate the hepatic "function". However, this technique is not instantaneously available and thus cannot been used intra-operatively (during liver surgery). Near-infrared spectroscopy enables to assess hepatic ICG concentration over time in the liver tissue. This article proposes to extract more information from the liver intensity dynamics by interpreting it through a dedicated pharmacokinetics model. In order to account for the different exchanges between the liver tissues, the proposed model includes three compartments for the liver model (sinusoids, hepatocytes and bile canaliculi). The model output dependency to parameters is studied with sensitivity analysis and solving an inverse problem on synthetic data. The estimation of model parameters is then performed with in-vivo measurements in rabbits (El-Desoky et al. 1999). Parameters for different liver states are estimated, and their link with liver function is investigated. A nonlinear (Michaelis-Menten type) excretion rate from the hepatocytes to the bile canaliculi was necessary to reproduce the measurements for different liver conditions. In case of bile duct ligation, the model suggests that this rate is reduced, and that the ICG is stored in the hepatocytes. Moreover, the level of ICG remains high in the blood following the ligation of the bile duct. The percentage of retention of indocyanine green in blood, which is a common test for hepatic function estimation, is also investigated with the model. The impact of bile duct ligation and reduced liver inflow on the percentage of ICG retention in blood is studied. The estimation of the pharmacokinetics model parameters may lead to an evaluation of different liver functions.
\end{abstract}

Keywords: Pharmacokinetics model; Sensitivity analysis; Parameter estimation; Indocyanine

\footnotetext{
${ }^{*}$ Corresponding author

Email addresses: chloe.audebert@inria.fr; +33(0)1.80.49.42.74 (Chloe Audebert), irene.vignon-clementel@inria.fr; +33(0)1.80.49.42.67 (Irene E. Vignon-Clementel)

* A final version of this manuscript can be found in European Journal of Pharmaceutical Sciences (2018)
} 
green fluorescence; Liver function estimation

\section{Introduction}

Liver function can be evaluated with blood sample analysis, quantifying the increase of biliru„bine for example. The indocyanine green (ICG) is a dye eliminated exclusively by the liver Alander et al. (2012); Hoekstra et al. (2013)). Therefore it is used for determining liver function. At the 5 moment, a reliable method to estimate global hepatic "function" is the ICG clearance, presented as plasma disappearance rate. In fact, this biomarker combines the effect of liver perfusion and functions. Blood analysis requires time and therefore is not instantaneously available during liver surgery. The liver surgeries (partial hepatectomy and liver transplantation) are the only curative treatments for advanced liver disease. The principal lethal complications after these surgeries are the post-operative liver failure, after major partial hepatectomy, and the small-for-size syndrome, after partial liver transplantation (Tucker and Heaton (2005)). Both complications are related to a poor pre- or intra-operative evaluation of the function of the remnant or transplanted liver. Therefore a tool for the intra-operatively assessment of liver function is interesting for the clinics.

Liver function can be divided into two steps: the uptake by the liver cells (the removal of the compound from the sinusoids blood) and the secretion from the cells into the bile. A good understanding of ICG processing by liver may improve the liver function assessment and help clinical decision. This article proposes to improve knowledge on ICG processing by the liver, in healthy and pathological situations, based on mathematical modeling and numerical simulations.

Several modeling works exist on transport and diffusion of various compounds (tracer, antibiotic, hormone) in the liver (Schwen et al. (2014)), using pharmacokinetics models and including the wholebody PBPK (physiologically based pharmacokinetics) (Schwen et al. (2015)), or for tumor detection with Magnetic Resonance Images modeling (Bezy-Wendling et al. (2007)). Recently, a compartment model that included metabolism of ammonia detoxification has guided the identification of a therapeutic strategy for liver disease (Ghallab et al. (2016)).

Regarding ICG, its concentration in the blood circulation has been studied based on pharmacokinetics models. A minimal physiological model for liver uptake and excretion rate estimation has been developed by (Weiss et al. (2011)), that includes compartments for pulmonary circulation, systemic circulation, gut and liver. Parameter estimation has been based on arterial ICG concentration over time in dogs that are awake and under anesthesia. The ICG clearance traditional sensitivity to sinusoidal clearance uptake and canalicular excretion rates has been studied. Since anesthesia tends 
to decrease cardiac output, the impact of a reduced flow on the estimated parameter has been also analyzed. Their results suggest that ICG uptake is limited by sinusoidal clearance uptake capacity and bile excretion, and not by blood flow. The ICG plasma disappearance rate has been largely studied. In particular, a two-compartment pharmacokinetics model validated on healthy liver has been proposed by (Grainger et al. (1983)). The same model has been subsequently studied for patients with liver cirrhosis, and a tri-exponential function to better describe the elimination of ICG from plasma has been proposed by (Burns et al. (1991)). Two fractions of ICG contained in dye preparations have been taken into account by (Ott et al. (1994)), suggesting that the two fractions disappearing rates are different. Measurements in pigs have been performed, and two types of model have been considered, including or not a temporary storage compartment to determine if taking into account extrahepatic, extravascular distribution is required to explain the measurements. A four compartments model has been proposed to explain measured plasma disappearance and biliary excretion of ICG in dogs chronically intoxicated with dimethylnitrosamine (Kawasaki et al. (1984)). Plasma to liver transfer rate constant was found significantly smaller in intoxicated animals. In all these models, the liver is represented with a single compartment, and therefore the exchanges between the different liver tissues are not modeled. The pharmacokinetics of ICG in cancerous tumor has been modeled by (Alacam et al. (2006)). After tumor cell injection under the skin of rats, the pharmacokinetics parameters have been estimated with ICG tumor tissue concentration measurements, using the extended Kalman filter. The exchange rate between blood and tissue was estimated, and edematous and necrotic tissue rates were compared. The model parameters may be useful for tumor differentiation.

Finally, the liver tissue ICG concentration has been less studied than plasma disappearing rate. Hepatic ICG concentration over time on rabbit liver has been measured with near-infrared spectroscopy (Shinohara et al. (1996); El-Desoky et al. (1999)). The measurement curves have been fitted with ${ }_{55}$ the sum of two exponential functions. Two parameters have been estimated, one interpreted as accounting for liver perfusion and dye uptake from plasma and a second for dye removal from the liver (accounting for bile secretion and bile flow). The changes of uptake and excretion parameters due to different treatments (colchicine, vessel occlusion ...) have also been analyzed (El-Desoky et al. (1999)).

This article focuses on a precise description of the ICG processing by the liver, including three compartments for the liver model (sinusoids, hepatocytes and bile canaliculi). Contrary to previous works, this model enables to precisely quantify the different exchanges between liver tissues, i.e. ICG 
uptake from sinusoids to hepatocytes and ICG excretion from hepatocytes to bile canaliculi. These exchanges are related to different liver functions, thus a precise quantification of the two exchanges may give information on the underlying pathology; e.g. portal thrombosis, which impedes the inflow to the liver, and bile duct obstruction are two pathologies impacting different functions of the liver. The model is based on ordinary differential equations and parameters are estimated with an inverse problem resolution based on the unscented Kalman filter. The model output sensitivity to parameters is studied and the inverse problem resolution is verified on synthetic data. Then, the estimation of model parameters is performed on the measurements from (El-Desoky et al. (1999) $)$ in rabbits. They are estimated for different liver states, and their link with liver function is investigated. The model predictions are then tested under different liver hypoperfusions, representing hepatic artery or portal vein inflow obstructions. The proposed model, its sensitivity to data and its validation are then discussed, along with ICG clinical aspects. This article proposes to extract more information from the liver ICG concentration dynamics than is currently done by interpreting it through a dedicated pharmacokinetics model. The estimation of the pharmacokinetics model parameters may lead to a finer evaluation of liver functions.

\section{Methods}

\subsection{Indocyanine green pharmacokinetics model}

The presented model aims at representing the transport of indocyanine green (ICG) (a fluorescent dye) in blood and its processing by the liver. After blood injection ICG exits the body exclusively through liver (Alander et al. (2012); Hoekstra et al. (2013)). The ICG goes through the liver, composed by mainly three types of tissue; a) blood vessels, in particular the sinusoids, which are the smallest blood vessels of the liver, where exchanges occur, b) hepatocytes, which are the liver cells, that take ICG from the sinusoids blood and secrete it into the bile, and c) bile canaliculi, small canals that transport bile to the common bile duct out of the liver.

To represent the ICG concentration dynamics over time, a pharmacokinetics model based on a compartmental structure is developed. The compartments are the sinusoids, the hepatocytes, the bile canaliculi and the rest of the blood circulation (Figure 1).

The compound is assumed to be injected in the rest of the blood circulation. Then, due to the double perfusion of the liver, ICG is transported in blood to the liver through the hepatic artery (HA) and the portal vein (PV), and reaches the hepatocytes through smaller vessels, the sinusoids. A passive exchange is assumed between sinusoids and hepatocytes. This exchange has 
been characterized by two parameters by (Weiss et al. (2011)). Assuming a passive exchange is similar to assuming that these two parameters are equal. ICG is secreted from the hepatocytes into the bile canaliculi, with a process assumed active and possibly saturating (De Gasperi et al. (2016)). The saturation is modeled by parameter $S$ in the following equations. If the product of $S$ and the concentration in the hepatocytes is significantly higher than 1, then the secreted amount of ICG is independent of the hepatocytes ICG concentration. The usual Michaelis Menten parameters $V_{\max }$ and $K_{m}$ can be expressed as a function of $S$ and $Q_{h b}$ parameters as follows: $V_{\max }=Q_{h b} /\left(V_{h} S\right)$ and $K_{m}=1 / S$. Our choice of parameters (which is unusual) is detailed and discussed in section 4 Then, if not taken by the hepatocytes ICG exits the liver through the hepatic veins and returns to the rest of the blood circulation, or it reaches the common bile duct, carried with the bile through the bile canaliculi and biliary tree. The liver ICG amount is assumed to be the sum of the amounts in sinusoids, hepatocytes and bile canaliculi compartments. Figure 1 shows a schematic representation of the model. Notation for parameters and concentrations are summarized in Table 1. The model described above is defined by the following equations

$$
\begin{cases}\frac{d}{d t}\left(V_{\text {blood }} C_{\text {blood }}\right) & =\left(F_{h a}+F_{p v}\right) C_{s}-\left(F_{h a}+F_{p v}\right) C_{b l o o d} \\ \frac{d}{d t}\left(V_{s} C_{s}\right) & =\left(F_{h a}+F_{p v}\right) C_{b l o o d}-\left(F_{h a}+F_{p v}\right) C_{s}-K_{s h}\left(C_{s}-C_{h}\right) \\ \frac{d}{d t}\left(V_{h} C_{h}\right) & =K_{s h}\left(C_{s}-C_{h}\right)-\frac{Q_{h b}}{1+S C_{h}} C_{h} \\ \frac{d}{d t}\left(V_{b c} C_{b c}\right) & =\frac{Q_{h b}}{1+S C_{h}} C_{h}-F_{b c} C_{b c}\end{cases}
$$

and the liver ICG amount is defined as follows

$$
V_{L} C_{L}=V_{s} C_{s}+V_{h} C_{h}+V_{b c} C_{b c}
$$

The state variables (dynamical model outputs) are thus chosen as the 4 component-vector $y=$ ( $V_{\text {blood }} C_{\text {blood }}, V_{s} C_{s}, V_{h} C_{h}, V_{b c} C_{b c}$ ) of the ICG amounts in each compartment.

The non-linear system of equations (1) - (2) is solved with the IDA package, a part of SUNDIALS (Suite of Nonlinear and Differential/Algebraic Equation Solver) (Hindmarsh et al. (2005)). The time-integration is done with the Backward Differentiation Formula (BDF) method; the non-linear algebraic system is then solved with a Newton method.

\subsection{Available data for parameter estimation}

(El-Desoky et al. (1999)) present typical indocyanine green curves of concentration over time in the rabbit liver. The goal here is to use these measurements to identify the model parameters. The 
Table 1: Notation and units for parameters and variables of the mathematical model.

\begin{tabular}{|c|c|}
\hline Notation & Physiological meaning \\
\hline blood & Rest of blood circulation \\
\hline $\mathrm{s}$ & Sinusoids \\
\hline $\mathrm{h}$ & Hepatocytes \\
\hline $\mathrm{bc}$ & Bile Canaliculi \\
\hline $\mathrm{L}$ & Liver \\
\hline$F_{h a}$ & Hepatic artery flow rate (Volume/Time) \\
\hline$F_{p v}$ & Portal vein flow rate (Volume/Time) \\
\hline$V_{i}$ & Volume of $i^{t h}$ compartment \\
\hline$C_{i}$ & Concentration of $i^{t h}$ compartment (arbitrary units AU) \\
\hline$F_{i}$ & $i^{\text {th }}$ flow rate (Volume/Time) \\
\hline$K_{s h}$ & Exchange coefficient between sinusoids and hepatocytes (Volume/Time) \\
\hline$Q_{h b}$ & Excretion coefficient from hepatocytes to bile canaliculi (Volume/Time) \\
\hline$S$ & Saturation parameter (1/Concentration; 1/AU) \\
\hline$V_{\max }$ & $\left(=Q_{h b} /\left(S V_{h}\right)\right)$ Michaelis Menten parameter for maximum rate at saturating concentration $(\mathrm{AU} / \mathrm{s})$ \\
\hline$K_{m}$ & $(=1 / S)$ Michaelis Menten parameter for concentration at which the rate is half-maximum (AU) \\
\hline
\end{tabular}




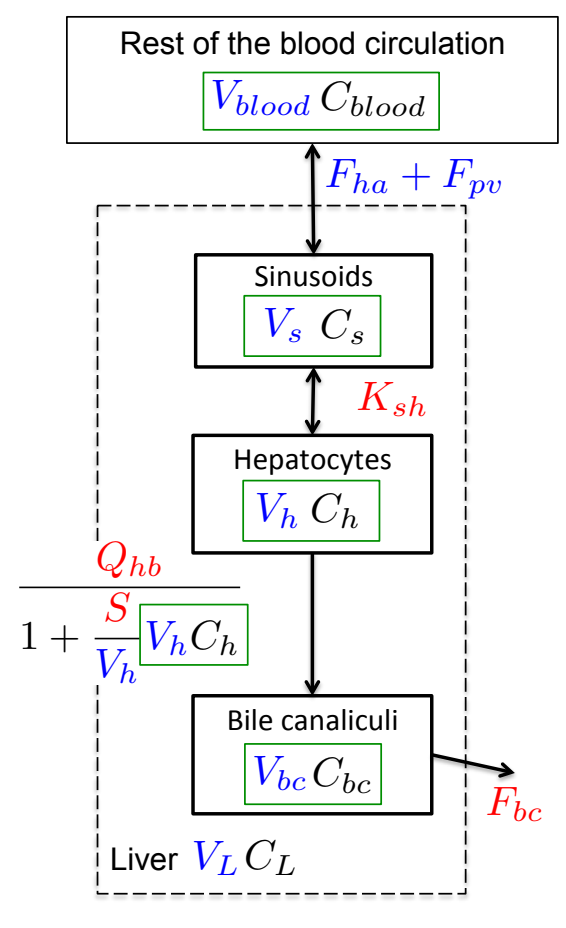

Figure 1: Model schematic description. The plain black boxes indicate the 4 compartments. The dash-box corresponds to the entire liver. The arrows indicate the direction of compound transfer. The parameters in blue are the ones fixed with literature data or non-ICG measurements, and in red are the estimated parameters by the solution of an inverse problem. The state variables are framed in green. 
parameters are not all estimated with the Unscented Kalman Filter: some are fixed from literature or from non-ICG measurements, as detailed below and summarized in Table 2

The curves from (El-Desoky et al. (1999)) are in arbitrary units. Thus, in this work, the amount unit is concentration arbitrary unit times volume in ml (AU.ml). (El-Desoky et al. (1999)) gave the injected ICG quantity. However, since the concentration functions are in arbitrary units, the bolus function is unknown. Here, the initial time $(t=0 \mathrm{~s})$ is assumed to be just after the bolus injection in the blood circulation. Therefore, a non-zero initial value for ICG amount in the blood compartment is assumed, and the ICG amount in the other compartments is set to zero. Finally, (El-Desoky et al. (1999)) measured the hepatic artery and portal vein flows. Hence the parameters $F_{h a}$ and $F_{p v}$ are known (Figure 1 in blue, Table 2).

All compartment volumes are fixed based on the literature as follows (Figure 1 in blue, Table 2). The total circulating volume is assumed to be $56 \mathrm{ml} / \mathrm{kg}$ of the animal body weight (Diehl et al. (2001)). The weight of the rabbit, considered by (El-Desoky et al. (1999)), is $2.9 \mathrm{~kg}$ in average. Therefore, the rabbit average blood volume is assumed equal to $160 \mathrm{ml}$. The rabbit liver weight is reported to be $2.7 \%$ of body weight by (Webster et al. (1947)). The liver is mainly composed of blood or water. Therefore, the liver mass density is chosen close to blood density $(1 \mathrm{~g} / \mathrm{ml})$. Finally, the rabbit liver volume estimation is $80 \mathrm{ml}$. (Hammad et al. (2014) ) reports, in a hepatic lobule, the sinusoids and the bile canaliculi volumes, in percentage of the tissue volume. The results are extended to the whole liver and the remaining volume is assumed to be the hepatocytes volume. The estimated volumes are then, for the sinusoids, $12.3 \mathrm{ml}$, for the hepatocytes $65 \mathrm{ml}$ and for the bile canaliculi $2.7 \mathrm{ml}$. The remaining parameters to estimate are $K_{s h}, Q_{h b}, S$ and $F_{b c}$ (Figure 1 in red).

(El-Desoky et al. (1999)) collected data on 36 rabbits, separated in 6 groups. Here, two of these groups are used for parameter estimation; the control group and the bile duct ligation group. ICG concentration time-curves are fitted for all groups with a sum of two exponential functions: $-A e^{-\alpha t}+B e^{-\beta t}$ (El-Desoky et al. (1999)). For the two parameters $\alpha$ and $\beta$ the mean and standard deviation are reported. Moreover, a typical curve (and only one) is drawn for each group. $A$ and $B$ can be identified from it. The curves from the paper start from non-zero values. In this work, the amount of ICG in the liver at time $t=0 \mathrm{~s}$ is assumed null, because the initial time is assumed to be just after bolus injection. Thus, no ICG has arrived to the liver yet. To obtain observation curves starting at zero, the curves from (El-Desoky et al. (1999)) are shifted in time. The shift in time does not change $\alpha$ and $\beta$ values, but new values for $\mathrm{A}$ and $\mathrm{B}=\mathrm{A}$ are found. The $\alpha$ and $\beta$ parameters 
Table 2: Fixed parameter values, from literature data or from non-ICG measurements reported by (El-Desoky et al. (1999)).

\begin{tabular}{|cc|}
\hline Parameter & Value \\
\hline$V_{\text {blood }}$ & $160 \mathrm{ml}$ \\
$V_{L}$ & $80 \mathrm{ml}$ \\
$V_{s}$ & $12.3 \mathrm{ml}$ \\
$V_{h}$ & $65 \mathrm{ml}$ \\
$V_{b c}$ & $2.7 \mathrm{ml}$ \\
$F_{h a}$ & $0.25 \mathrm{ml} / \mathrm{s}$ \\
$F_{p v}$ & $1.5 \mathrm{ml} / \mathrm{s}$ \\
\hline
\end{tabular}

150 also considered. Generalized sensitivity functions analysis reveals important correlation between parameters, and the distribution over time of information on a parameter contained into the observations. Generalized sensitivity functions start at value zero and end at one. The increase is not 

The time interval, where the sharpest increase appears in the generalized sensitivity function, is where most information on the parameter is contained during the observations (Bai et al. (2007)).

In this work, the sensitivity functions are computed with the IDAS solver, which is a part of SUNDIALS (Hindmarsh et al. (2005)). The two sensitivity tools are local analyses: the initially chosen parameters impact the sensitivity functions. Therefore, the sensitivity analysis is performed for two different sets of parameters, representing two regimes of the dynamical system. The sensitivity of ICG amount in the liver compartment (equation (2p) to the parameters $K_{s h}, Q_{h b}, S$ and $F_{b c}$ is studied. The other parameters are set as explained in section 2.2. The initial conditions are: in the blood compartment $V_{\text {blood }} C_{\text {blood }}=83000 \mathrm{AU} . \mathrm{ml}$ and set to zero in the other compartments. Two different parameter sets are studied to consider different types of observation curve.

In the first case, a fast decrease of ICG in the liver occurs (Figure 2 (a) left), due to a low saturation parameter S. The first parameter set of the synthetic observation curve is: $K_{s h}=1.0$ $\mathrm{ml} / \mathrm{s}, Q_{h b}=6.5 \mathrm{ml} / \mathrm{s}, S=0.0011 / \mathrm{AU}$ and $F_{b c}=0.05 \mathrm{ml} / \mathrm{s}$. In the second case, a higher saturation parameter is chosen ( $S=0.21 / \mathrm{AU}$ ), leading to a slower decrease of liver ICG amount (Figure 2 (b) left). All the other parameters are the same. The two cases correspond to two different regimes :

- Case 1: $S C_{h}<<1$, the exchange coefficient $\frac{Q_{h b}}{1+S C_{h}} C_{h}$ is similar to $Q_{h b} C_{h}$ (linear in $C_{h}$, so first-order kinetics)

- Case 2: $S C_{h}>>1$, the exchange coefficient $\frac{Q_{h b}}{1+S C_{h}} C_{h}$ is similar to $Q_{h b} / S$ (independent of $C_{h}$, , i.e. zero-order kinetics)

The generalized sensitivity functions are plotted by pairs, assuming the other parameters are known. Analyzing generalized sensitivity functions two by two enables to better understand the correlations between parameters.

\subsection{Parameter estimation method}

Sensitivity analysis informs about parameter estimation problem difficulties, according to available measurements. Once the set of parameters to be reasonably estimated is defined, an algorithm to find the parameter values based on the available measurements also needs to be selected. The Unscented Kalman Filter (UKF) algorithm is here chosen for parameter estimation (see (Julier et al. (1995)) for method details).

The Kalman filter is initially a state estimator for linear dynamical systems. For non-linear 200 systems various modifications of the Kalman filter have been proposed, e.g. the extended Kalman 
filter and the unscented Kalman filter (Haykin et al. (2001); Simon (2010)). The Unscented Kalman Filter (UKF) algorithm (Julier et al. $(1995,2000)$ ) has been used for state and parameter estimation for different dimensional systems (Moireau and Chapelle (2011); Bertoglio et al. (2012); Pant et al. (2014, 2017)). The UKF algorithm consists of two steps: the state mean and covariance are propagated in time, by solving the model equations, and the state mean and covariance are then corrected with the noisy measurements (model output transformation, called observations). Here, the forward model is fast and easy to solve. Therefore the UKF algorithm technique is well-adapted. The parameter estimation, in UKF, is based on a prior confidence. The two coefficients, $\sigma_{o b s}$ and $\sigma_{\text {init }}$, are standing for the confidence in the measurements and in the initial guess respectively. These two coefficients may impact the UKF results. Therefore, in the next section, different initial guesses are considered with varying confidence. The parameter estimation method is used to estimate parameters from synthetic data (model observation with added noise) and from in-vivo data extracted from (El-Desoky et al. (1999)).

Synthetic data are generated by choosing a set of parameters and solving the model equations (the direct problem (1) - (2)). The resulting amount in the liver, with added noise, can thus be taken as a synthetic observation. If the inverse procedure works well, one should recover as estimated parameters the parameter set used for the direct problem (called true parameter). This procedure enables to verify the method as well as inform on parameter identifiability issues. Similarly to sensitivity analysis, two sets of parameters were chosen to generate two synthetic curves that correspond to two different regimes. A Gaussian noise of zero mean and with a given variance is added to the direct problem solution (the given variance corresponds to $\sigma_{o b s}^{2}=0.02$ ). The noise variance for the synthetic cases is chosen to be the same as for the in-vivo data case, i.e. to mimic the level of noise observed by (El-Desoky et al. (1999)). For all the inverse problems, the UKF algorithm is performed three times in a row. First, UKF is performed with the initial guess and the confidence 225 parameter $\sigma_{\text {init }}^{2}=1.0$. Then, UKF is run again with the estimation of the first run as initial guess and a reduced confidence $\sigma_{\text {init }}^{2}=0.5$. Finally, the UKF is run a third time, with the estimates of the second run and the confidence is decreased $\sigma_{\text {init }}^{2}=0.3$.

\section{Results}

In the next sections, the sensitivity of the liver amount (equation (2)) to the parameters of interest is analyzed. Then, to verify the approach, the inverse problem is solved with noisy synthetic data as observation (meaning that the data are generated by the model itself, adding noise). The 
sensitivity analysis and the inverse problem are performed for two different regimes of the dynamical system. Finally, measurement curves from (El-Desoky et al. (1999)) are the basis for estimating the parameters of interest. known. $S$ and $Q_{h b}$ GSFs present much higher values and oscillations (25 times) more than in the 

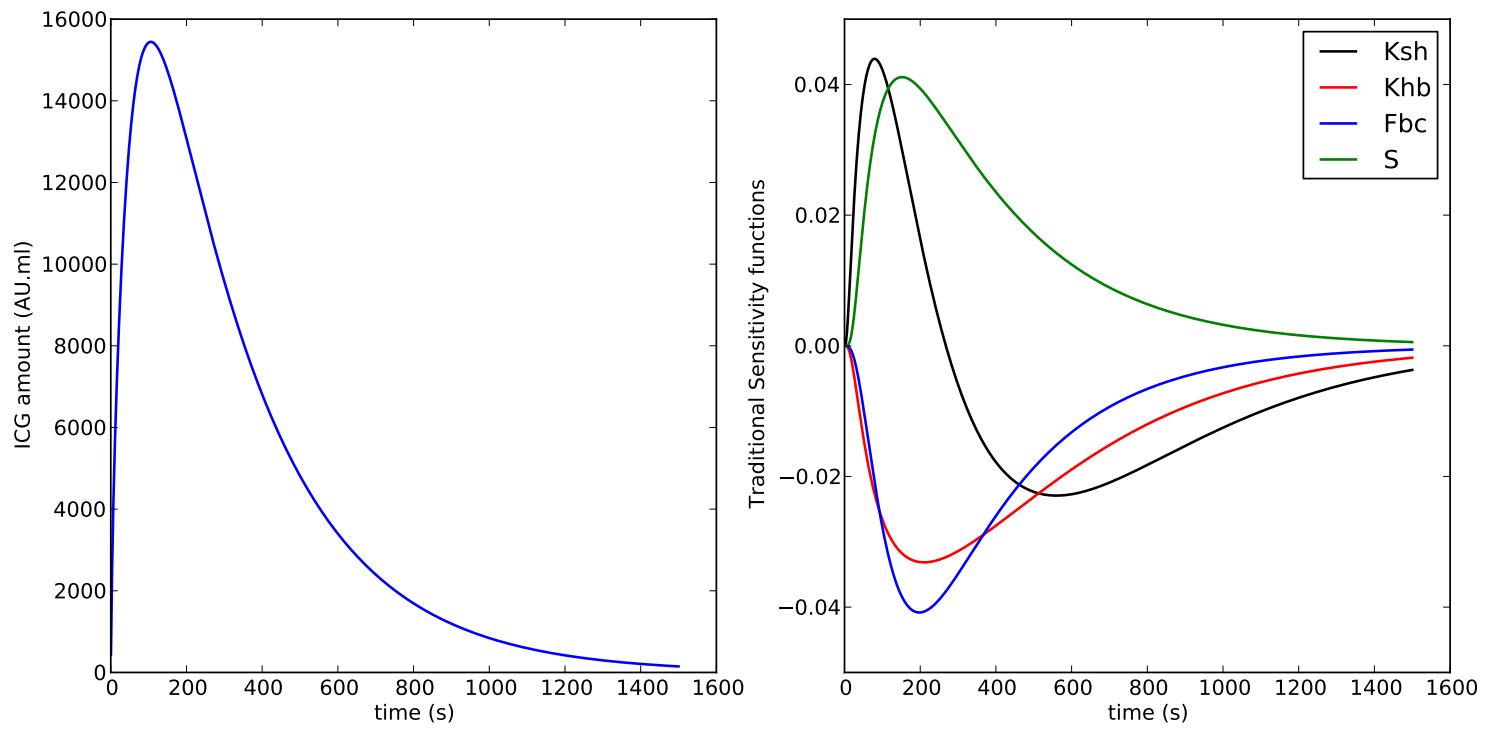

(a) Case 1
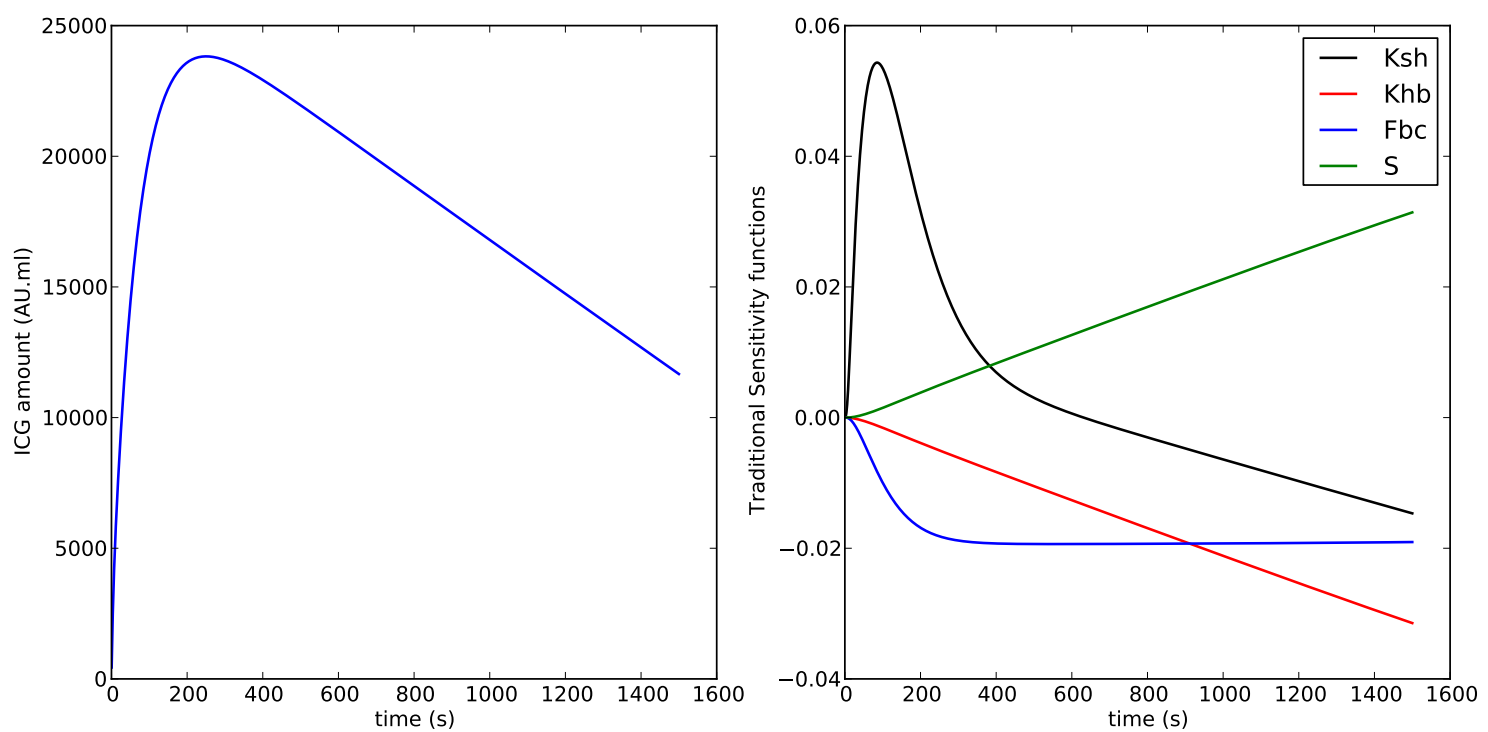

(b) Case 2

Figure 2: Traditional sensitivity function. Left: ICG amount in the liver over time. Right: Traditional sensitivity functions for $K_{s h}, Q_{h b}, S$ and $F_{b c}$ parameters. 

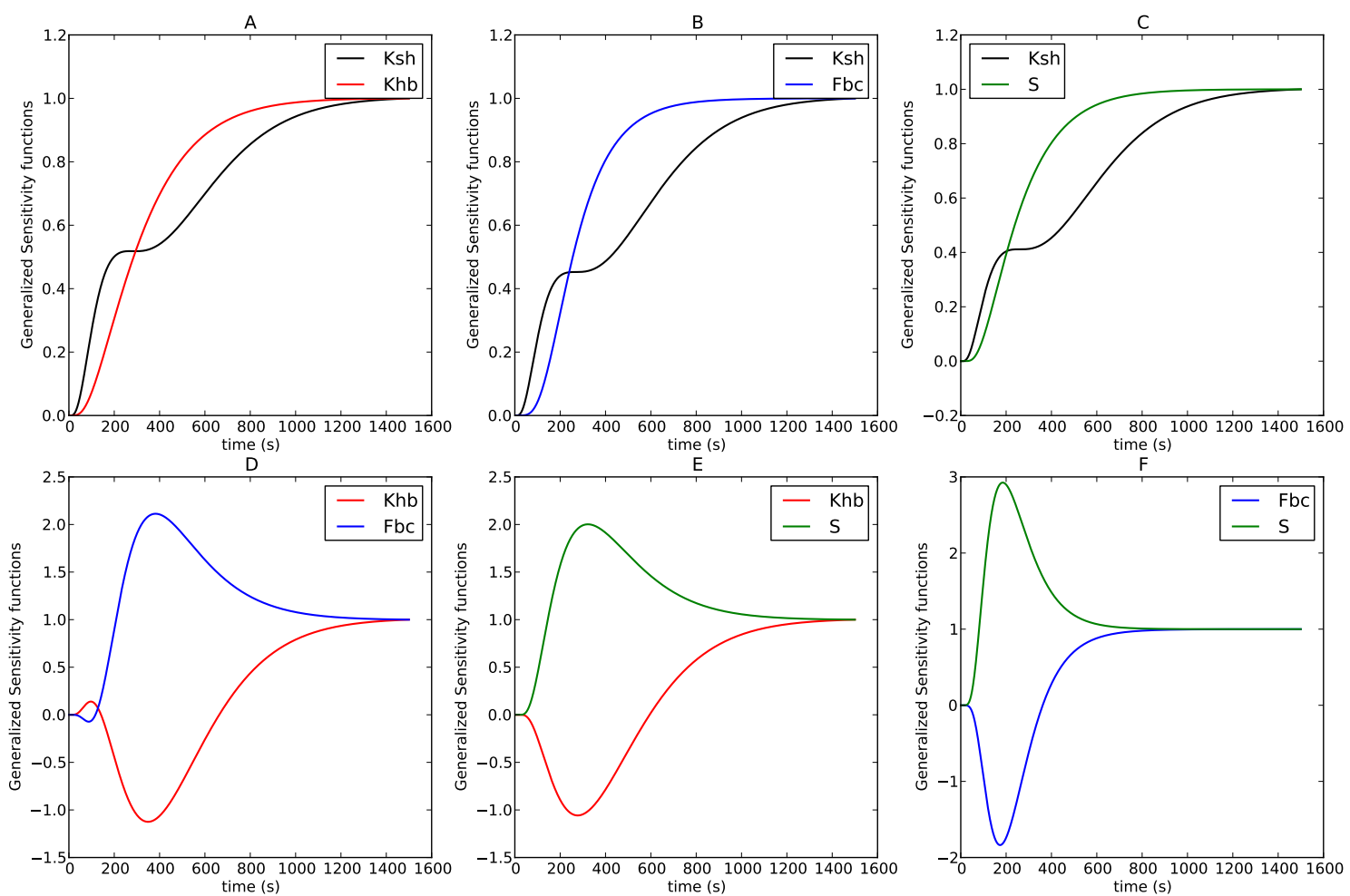

Figure 3: Case 1: Generalized sensitivity functions. Generalized sensitivity functions for two parameters, with the two other parameters fixed, for all pairs of parameters $K_{s h}, Q_{h b}, \mathrm{~S}$ and $F_{b c}$. A: $F_{b c}$ and $S$ are fixed; B: $Q_{h b}$ and $\mathrm{S}$ are fixed; C: $Q_{h b}$ and $F_{b c}$ are fixed; D: $K_{s h}$ and S are fixed; E: $F_{b c}$ and $K_{s h}$ are fixed; F: $K_{s h}$ and $Q_{h b}$ are fixed. 

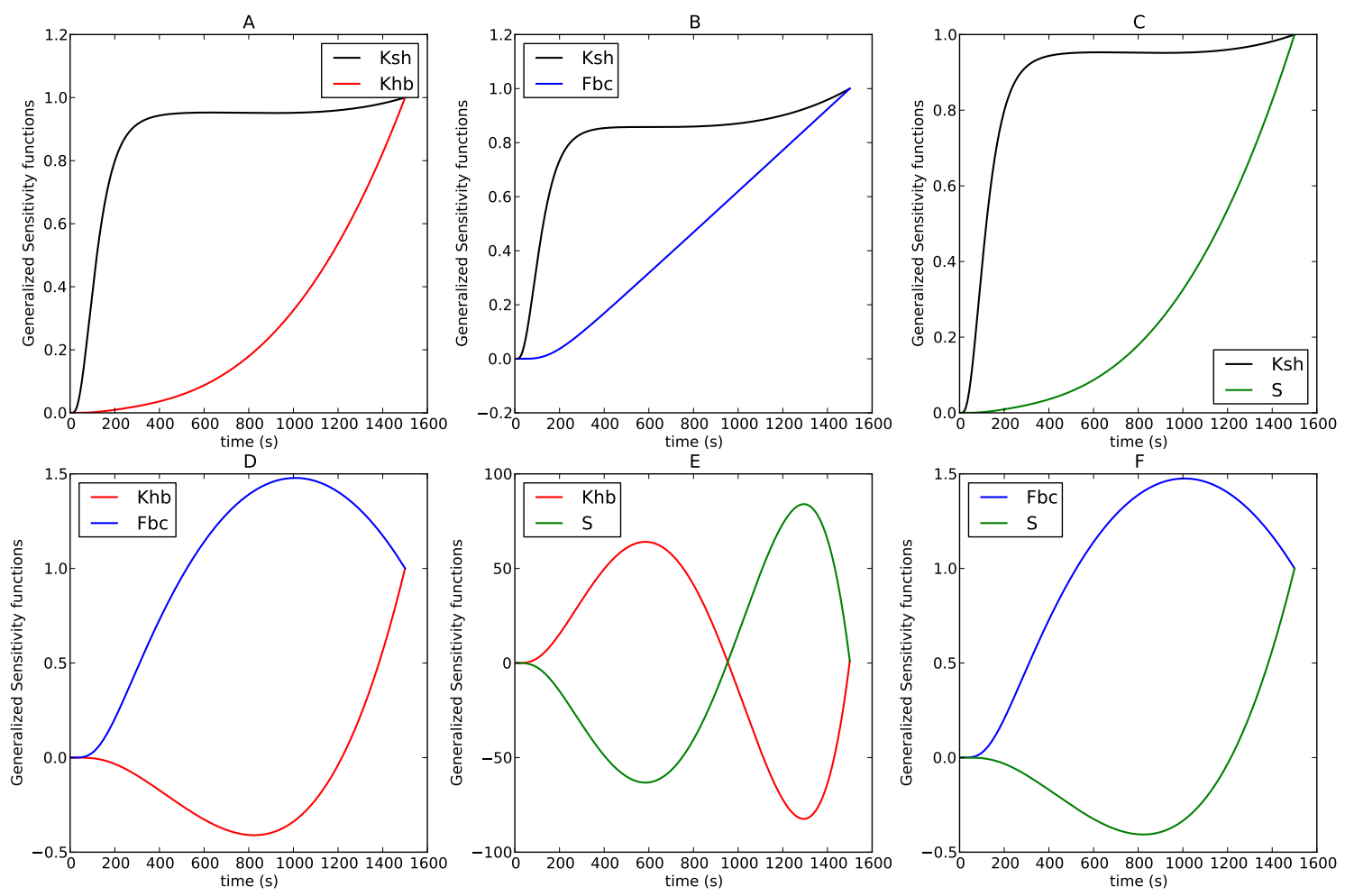

Figure 4: Case 2: Generalized sensitivity functions. Generalized sensitivity functions for two parameters, with the two other parameters fixed, for all pairs of parameters $K_{s h}, Q_{h b}, \mathrm{~S}$ and $F_{b c}$. A: $F_{b c}$ and $S$ are fixed; B: $Q_{h b}$ and $\mathrm{S}$ are fixed; C: $Q_{h b}$ and $F_{b c}$ are fixed; D: $K_{s h}$ and S are fixed; E: $F_{b c}$ and $K_{s h}$ are fixed; F: $K_{s h}$ and $Q_{h b}$ are fixed.

previous case (Figure 4 E). Such GSFs reveal large correlations between the two parameters. Figure $4 \mathrm{D}$ and $\mathrm{F}$ reveal small correlations and no oscillations appear in Figure 4 A, B and C.

The sharp increase of GSF suggests that, in the curve of liver ICG amount, most information, on $K_{s h}$, is contained in the increasing part of the curve, and the second part of the curve informs on the S, $Q_{h b}$ and $F_{b c}$ parameters.

In summary, the two cases have different sensitivity functions. However for both of them, $Q_{h b}$ and $S$ parameters are likely to be correlated (much more in the second case). Moreover, in the observations curve, most information about $K_{s h}$ is contained in the increasing part, and the decreasing part contains information about the other parameters. 
Table 3: Initial guess, true parameter and final parameter estimations for the different inverse problems performed in the first case (each time after three consecutive runs of UKF). The confidence in the estimation is also reported. Number of estimated parameters: 4 for the 1st estimation; 3 for the 2 nd and 3rd estimations.

\begin{tabular}{|c|c|c|c|c|}
\hline Parameter & $K_{s h}(\mathrm{ml} / \mathrm{s})$ & $Q_{h b}(\mathrm{ml} / \mathrm{s})$ & $\mathrm{S}(1 / \mathrm{AU})$ & $F_{b c}(\mathrm{ml} / \mathrm{s})$ \\
\hline Initial guess & 10 & 65 & 0.01 & 0.5 \\
\hline \multirow[t]{2}{*}{ True parameter } & 1.0 & 6.5 & 0.001 & 0.05 \\
\hline & \multicolumn{4}{|c|}{ First estimation } \\
\hline \multirow[t]{2}{*}{ Final estimate } & $0.87 \pm 0.0001$ & $782[635,963]$ & $4.21[3.32,5.19] \times 10^{-6}$ & $0.048 \pm 0.0001$ \\
\hline & \multicolumn{4}{|c|}{ Second estimation } \\
\hline \multirow[t]{2}{*}{ Final estimate } & $0.98 \pm 0.001$ & $7.8[7.6,8]$ & $0.007[0.006,0.008]$ & known \\
\hline & \multicolumn{4}{|c|}{ Third estimation } \\
\hline Final estimate & known & $6.52 \pm 0.001$ & $0.005[0.004,0.006]$ & $0.052 \pm 0.0001$ \\
\hline
\end{tabular}

\subsection{Parameter estimation on noisy synthetic data}

The inverse problem on synthetic data is performed to verify the approach and to confirm the shown in Figure 10 (see Appendix). Table 3 summarizes the final estimated values as well as the confidence in the estimation. $K_{s h}$ and $F_{b c}$ are well estimated (Appendix, Figure $10 \mathrm{C}$ and F), while $\mathrm{S}$ and $Q_{h b}$ are poorly estimated (Appendix, Figure $10 \mathrm{D}$ and $\mathrm{E}$ ). The direct model equations are then solved, fixing the parameters with UKF final estimates (Appendix, Figure 10 B) and compared to the original curve: the observation curve is not perfectly fitted.

Then, the same procedure is repeated, assuming the bile canaliculi flow parameter $\left(F_{b c}\right)$ is known. The final estimated values are presented in Table 3 (second estimation line). The results of the inverse problems are shown in Figure 11 (see Appendix). For all parameters, the converged value is close to the known value. Moreover, the observation curve is well fitted (Appendix, Figure 11B). Parameter

Now, assuming the parameter $K_{s h}$ is known, the other parameters are estimated with the same 
Table 4: Initial guess, true parameter and final parameter estimations for the different inverse problems performed in the second case (each time after three runs of UKF). The confidence in the estimation is also reported. Number of estimated parameters: 4 for the 1st estimation; 3 for the 2 nd and 3 rd estimations.

\begin{tabular}{|c|c|c|c|c|}
\hline Parameter & $K_{s h}(\mathrm{ml} / \mathrm{s})$ & $Q_{h b}(\mathrm{ml} / \mathrm{s})$ & $\mathrm{S}(1 / \mathrm{AU})$ & $F_{b c}(\mathrm{ml} / \mathrm{s})$ \\
\hline Initial guess & 10 & 65 & 0.01 & 0.5 \\
\hline \multirow[t]{2}{*}{ True parameter } & 1.0 & 6.5 & 0.2 & 0.05 \\
\hline & \multicolumn{4}{|c|}{ First estimation } \\
\hline \multirow[t]{2}{*}{ Final estimate } & $1.1 \pm 0.01$ & $9.81[9.8,9.83] \times 10^{5}$ & $4.5[3.7,5.5] \times 10^{-4}$ & $7.4[6.4,8.6] \times 10^{4}$ \\
\hline & \multicolumn{4}{|c|}{ Second estimation } \\
\hline \multirow[t]{2}{*}{ Final estimate } & $1.0 \pm 0.001$ & $18.4[16.6,20.3]$ & $0.58[0.52,0.64]$ & known \\
\hline & \multicolumn{4}{|c|}{ Third estimation } \\
\hline Final estimate & known & $5.9[5.4,6.6]$ & $0.18[0.16,0.2]$ & $0.052 \pm 0.001$ \\
\hline
\end{tabular}

procedure. Estimated parameters are close to the known values (Table 3 , third estimation line). The results are shown in Figure 12 (see Appendix). Parameter $S$ is overestimated and the confidence in this parameter is small in relative terms (the grey zone in Figure 12 E remains large over time, Appendix). The observation curve is however well fitted (Appendix, Figure 12 B).

Thus, with noisy synthetic observations (measurements), the four parameters cannot be estimated. However if $K_{s h}$ or $F_{b c}$ are known the other parameters are estimated such that the noisy measurement is well matched.

Case 2. The second regime of the dynamical system is now studied with the same procedure. First, the inverse problem is performed to estimate all parameters (Appendix, Figure 13). Table 4 summarizes the final estimated values and the confidence in the estimates. The estimated parameter $K_{s h}$ is close to the known value, while $Q_{h b}$ and $F_{b c}$ estimates explode (Figure $13 \mathrm{D}$ and F). Moreover, the $S$ parameter is poorly estimated (Figure $13 \mathrm{E}$ ). The observation curve is not correctly fitted as shown in Figure 13 B (Appendix).

When $F_{b c}$ is known, the inverse problems results are shown in Figure 14 (Appendix) and Table 4 sumarizes the estimated parameter values. $K_{s h}$ is well estimated. However, $Q_{h b}$ and $S$ are overestimated. Computing the ratio $\frac{Q_{h b}}{S}$, for the estimates and the known values, the following results are obtained

$$
\left(\frac{Q_{h b}}{S}\right)_{\text {estimate }}=31.7 ; \quad\left(\frac{Q_{h b}}{S}\right)_{\text {known }}=32.5
$$

From the sensitivity analysis, the parameter $Q_{h b}$ and $S$ are expected to be correlated. The ratio of 
the two estimated parameters is close to the known values ratio. Moreover, the observation curve is well fitted, even with the overestimated parameters $Q_{h b}$ and $S$ (Appendix, Figure 14 B). Therefore, in this regime, it is likely that the observation is sensitive to the ratio $\frac{Q_{h b}}{S}$, when $F_{b c}$ is known.

Finally, $K_{s h}$ is fixed, and estimation of the other parameters is performed with the same procedure; three runs of UKF algorithm (Appendix, Figure 15). $Q_{h b}$ and $S$ estimations are a little underestimated but converge to close values of the known parameters (Table 44. $F_{b c}$ is correctly estimated (Table 4) and the observation curve is well fitted (Appendix, Figure 15B).

As for the first regime, with noisy synthetic observation the four parameters cannot be estimated and the observation curve is mismatched. When $F_{b c}$ or $K_{s h}$ are known, then the parameter estimation is improved and the observations curve is well matched. When $F_{b c}$ is known, the unsatisfactory estimation of $S$ and $Q_{h b}$ but the good estimation of their ratio may be explained by the high correlation between these two parameters in this regime, as shown by the sensitivity analysis.

In summary, according to the inverse problems on synthetic observation and to the sensitivity analysis, the estimation of the four parameters is unlikely with the curve of ICG amount in the liver. However, fixing $F_{b c}$ or $K_{s h}$ leads to parameter estimation that results in well fitted observation curves. The estimated parameters have the correct order of magnitude, however they are given within a certain confidence and especially in the 2nd case, the set of parameters is not necessary the original one. This shows that more than one parameter set can lead to model output within the noise range of the measurement. In the next section the model parameters are estimated using two curves from in-vivo data (El-Desoky et al. (1999)): one curve belongs to the second case regime (bile duct ligation group) while the second curve is similar to the first case regime curve (control group). To estimate the model parameters for the bile duct ligation group curve the parameter $F_{b c}$ is fixed and for the control group, the parameter $K_{s h}$ is fixed based on the estimation from the bile duct ligation group.

\subsection{Parameter estimation on real data}

According to the results of the inverse problem on noisy synthetic data, the four parameters are unlikely to be all estimated. Therefore, first the parameters $K_{s h}, Q_{h b}$, and $S$ are estimated with the bile duct ligation group curve. Indeed, for this group, the bile canaliculi flow rate is known; it is equal to zero.

To estimate the initial amount of ICG in blood compartment, several UKF algorithm runs are performed, with the bile duct ligation group curve as observation. $F_{b c}=0 \mathrm{ml} / \mathrm{s}$, thus no ICG exits the 
system, and the sum of ICG amounts in all compartments at the end of the simulation should be equal to the initial injected amount. After several runs of UKF, the value $V_{\text {blood }} C_{\text {blood }}(t=0)=82960$ AU.ml is found. This value is used for the rest of the simulations.

The aim of this section is to obtain model parameters for both control and bile duct ligation groups and to compare them. For the bile duct ligation group, the parameter $F_{b c}$ is known $\left(F_{b c}=0\right.$ $\mathrm{ml} / \mathrm{s}$ ). The parameter $K_{s h}$ is assumed not impacted by the bile duct ligation, so the two groups share the parameter value $K_{s h}$. Under these assumptions, the estimated parameters differing between the two groups are $S$ and $Q_{h b}$. The parameter estimation procedure is the following:

1. Based on bile duct ligation group observation curve (with three runs of UKF), the parameters $K_{s h}, Q_{h b}, S$ are estimated.

2. The parameter $K_{s h}$ is fixed with the estimated value in 1 . Then, based on control group observation curve (with three runs of UKF), the parameters $Q_{h b}, S, F_{b c}$ are estimated.

According to the synthetic data examples (and the sensitivity analysis) more than one set of parameters lead to a well fitted noisy observation curve. To determine whether bile duct ligation impacts the parameters, the procedure to estimate both group parameters is repeated 100 times with different UKF initial guesses. The idea is to estimate ranges of parameters for both groups, and determine if these ranges are distinct. The initial guesses are uniformly sampled in the following intervals, [10,100] for $K_{s h},[0.01,1]$ for $Q_{h b},[0.001,1]$ for $S$ and $[0.001,0.01]$ for $F_{b c}$. Finally, as $K_{s h}$ is shared by both groups for each estimation procedure only $S$ and $Q_{h b}$ are estimated separately for each group. This procedure leads to several sets of parameter per group. Parameters $S$ and $Q_{h b}$ are related to the more commonly used Michaelis Menten parameters $V_{\max }$ and $K_{m}$. The latter are compared between the two groups. Figure 5 displays the model simulations run with the different estimated parameter sets. The bile duct ligation observation curve is reproduced with the different sets of parameters. Two groups of simulated curve appear for the control group: the first group captures the first part of the observation curve better while the second captures better the last part of the observation curve. Figure 6 (top) shows the histogram of the final estimates for $K_{s h}$ (estimated with the bile duct ligation group curve) and for $F_{b c}$ (estimated with the control group curve). The estimated $K_{s h}$ parameter is mostly between $12 \mathrm{ml} / \mathrm{s}$ and $20 \mathrm{ml} / \mathrm{s}$ (Figure6 top left) or smaller than $2 \mathrm{ml} / \mathrm{s} . F_{b c}$ is estimated, smaller than $0.5 \mathrm{ml} / \mathrm{s}$ or between $1 \mathrm{ml} / \mathrm{s}$ and $1.5 \mathrm{ml} / \mathrm{s}$ (Figure 6 top right). Both parameters $V_{\max }$ and $K_{m}$ are larger in the control group compared with the bile duct ligation estimation (Figure 6 bottom). In the control group two ranges of parameters appear: 

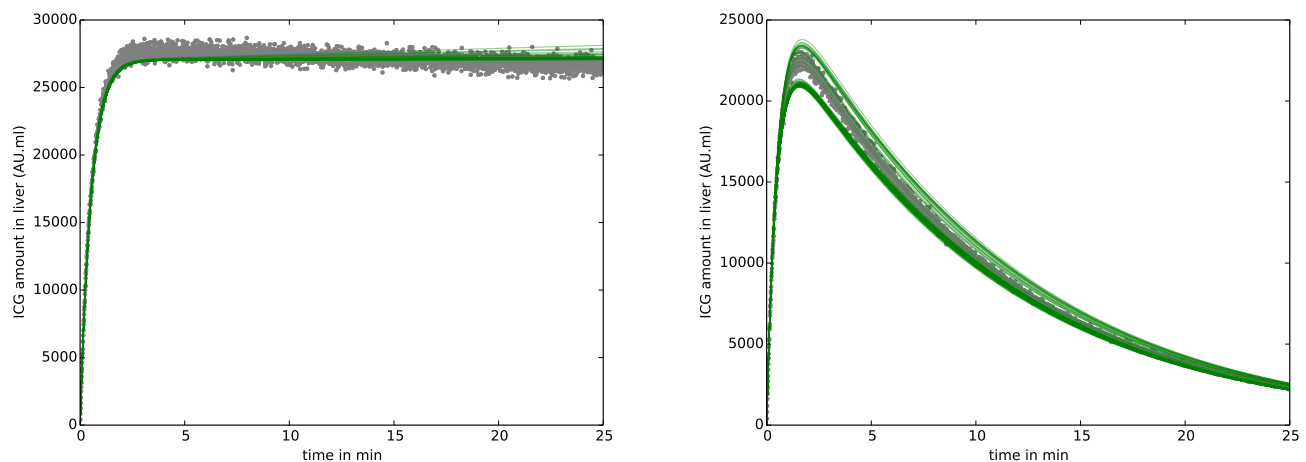

Figure 5: Measurement and model liver ICG amounts over time for the duct ligation group (left) and control group (right). Groups data curve from (El-Desoky et al. (1999)) with added noise (grey dots), and forward problem solutions (solid lines; one line per acceptable set of parameters found by the UKF algorithm).

$V_{\max }$ is mostly smaller than $1 \mathrm{AU} / \mathrm{s}$ or between $8 \mathrm{AU} / \mathrm{s}$ and $14 \mathrm{AU} / \mathrm{s}$; and $K_{m}$ is between $1 \mathrm{AU}$ and $5 \mathrm{AU}$ or $1000 \mathrm{AU}$ and $2000 \mathrm{AU}$. For bile duct ligation, parameter ranges are lower: $V_{\max }$ is smaller than $0.018 \mathrm{AU} / \mathrm{s}$ with two groups of values, one smaller than $0.004 \mathrm{Au} / \mathrm{s}$ and the second between $0.014 \mathrm{AU} / \mathrm{s}$ and $0.016 \mathrm{AU} / \mathrm{s} . K_{m}$ is distributed like a Gaussian with most values between $0.5 \mathrm{AU}$ and $1.5 \mathrm{AU}$.

To qualitatively compare the different behaviors, one of the estimated sets of parameters is chosen in each group. The control group parameters are $K_{s h}=13.8 \mathrm{ml} / \mathrm{s}, Q_{h b}=0.47 \mathrm{ml} / \mathrm{s}, S=$ $0.00077 A U^{-1}, F_{b c}=4.3 \mathrm{ml} / \mathrm{s}$, and for bile duct ligation group the parameter set is $K_{s h}=13.8 \mathrm{ml} / \mathrm{s}, Q_{h b}=$ $0.28 \mathrm{ml} / \mathrm{s}, S=2.6 \mathrm{AU}^{-1}, F_{b c}=0.0 \mathrm{ml} / \mathrm{s}$. Finally, two more pathophysiological conditions from (ElDesoky et al. (1999)) are used to further challenge the model: the hepatic artery (HA) occlusion group and portal vein (PV) partial occlusion group. The four parameters are set to be the same as for the control set, and simulations with various inflows are performed. The liver inflow is decreased by $10 \%$ (corresponding to HA occlusion), by $25 \%$, by $40 \%$ (corresponding to PV partial occlusion), by $70 \%$ and by $90 \%$. The percentage of occlusion corresponding to HA and PV are computed with the flow measurements reported in (El-Desoky et al. (1999)). The model solutions are plotted in Figure 7. along with the curve averages and standard deviations in the group of rabbits from (El-Desoky et al. (1999)). The model simulation global behavior is similar to the curves from the paper. Indeed, compared to the control group, when the liver inflow is decreased (HA occlusion), the maximum amount of ICG is reduced (Figure $7 \mathrm{C}$ ). Moreover, the time-to-peak (time at maximum value), is increased. However, the large standard deviation of the portal vein partial occlusion group in the 

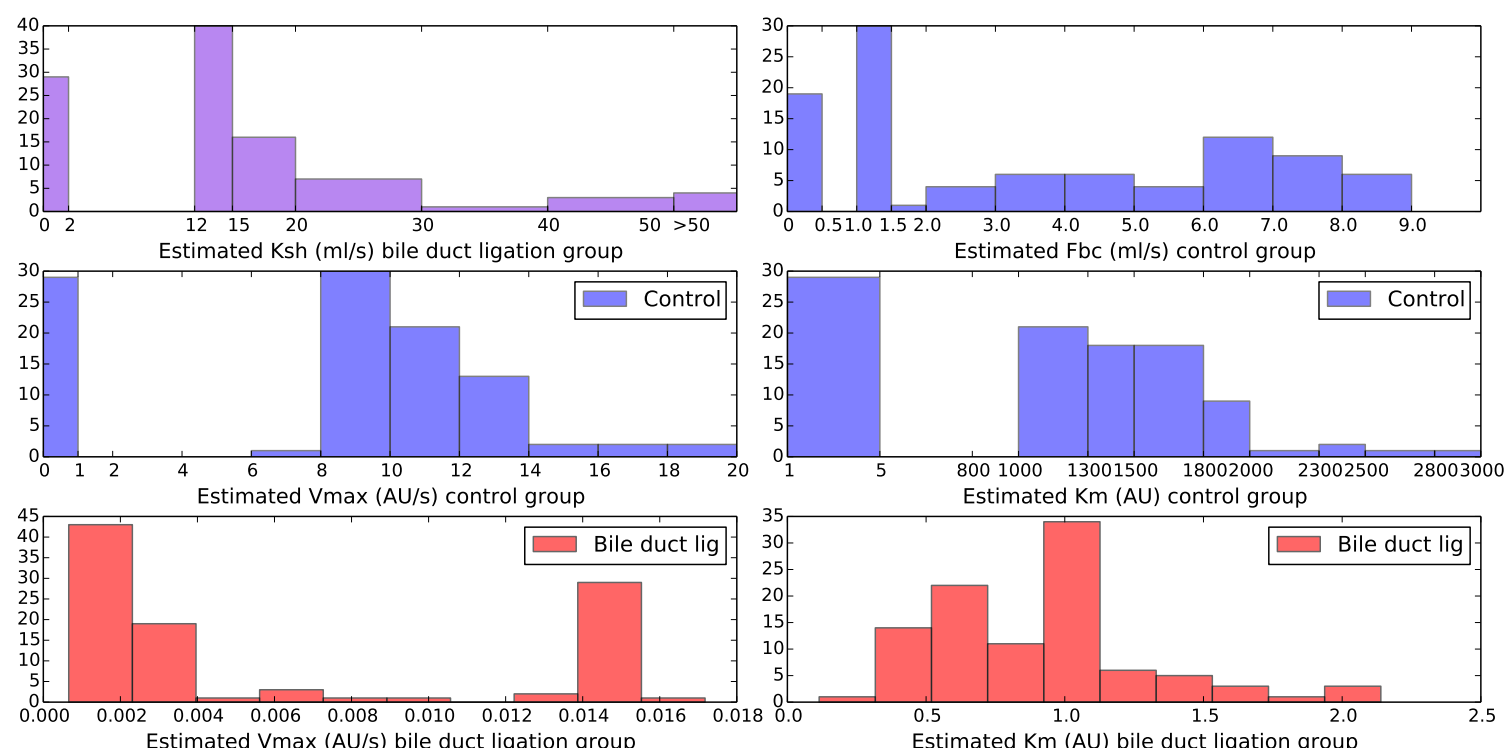

Figure 6: Histograms of parameters for the two groups, estimated with different initial guesses. Top left: $K_{s h}$ parameters, estimated with the bile duct ligation curve (parameter shared by both groups). Top right: $F_{b c}$ parameters, estimated with the control group curve. Comparison of estimated parameter ranges between the two groups: $V_{\max }$ (left) and $K_{m}$ (right) parameters, estimated with control (top blue) and bile duct ligation (bottom red) curves. 

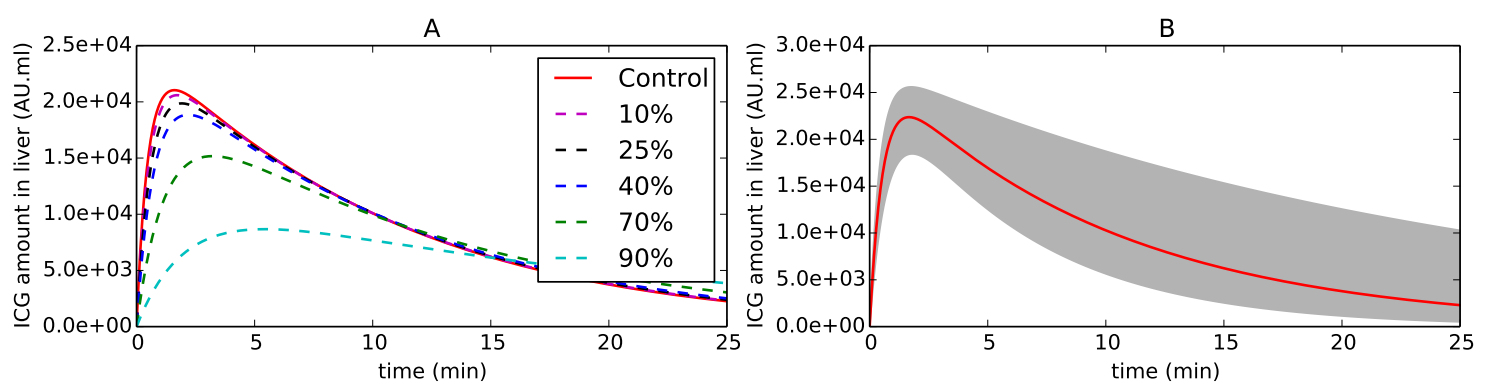

$\mathrm{C}$
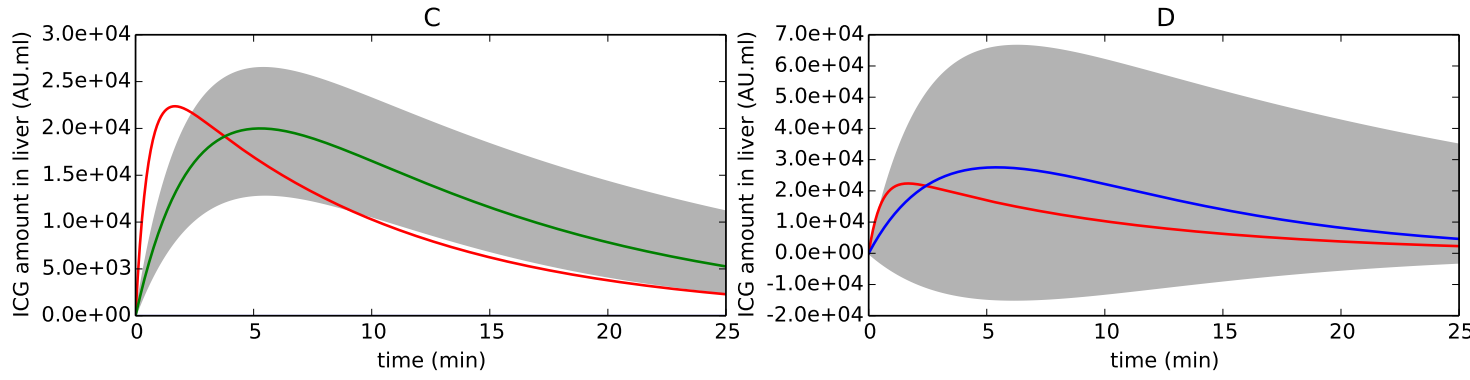

Control average measure - HA occlusion average measure

PV occlusion average measure

Figure 7: Model outputs and experimental observations for different liver inflows. A: ICG amount over time obtained with the model for different inflows; B: Control group average measure (solid line red) +/- standard deviation (grey zone) and control simulation (solid line black); C: Hepatic artery occlusion (solid black line) group average measure +/- standard deviation (grey zone) and control group average measure (solid red line); D: Portal vein partial occlusion (solid black line) group average measure +/- standard deviation (grey zone) and control group average measure (solid red line). The measurements are from (El-Desoky et al. (1999)). analyze.

\section{Discussion and conclusions}

A mathematical model has been developed for the indocyanine green processing by the liver. The liver amount sensitivity to parameters has been studied, and the inverse problem of estimating model parameters from liver dynamical data has been performed.

The mathematical model. In the model, some parameters is fixed from the literature. Others, such as the inflows, are set based on the experimental data reported in (El-Desoky et al. (1999)). The core parameters are then estimated from liver dynamical measurements, one curve for each pathophysiological group. The experimental measurements are well fitted with the proposed model. The 
400 liver than previously. In the model proposed by (Shinohara et al. (1996)) and used by (El-Desoky et al. (1999)), a single compartment describes the liver. Furthermore, that model does not take into account a re-circulation of ICG through the hepatic veins. However, the measurements from $\mathrm{Ott}$ et al. (1994)) have confirmed that ICG exits the liver through the hepatic veins. The re-circulation is taken into account in the model proposed by (Weiss et al. (2011)) and the complete blood circulation has been considered. However, the liver is then represented by a single compartment and the available data are ICG concentration in arterial blood samples, contrary to the liver tissue ICG concentration used here.

The three compartments in the liver are required to take into account the two exchanges of different nature: the exchange between sinusoids and hepatocytes, where no energy consumption is assumed, and the exchange between hepatocytes and bile canaliculi, where the transport is active, with possible saturation. The simple passive exchange between sinusoids and hepatocytes is enough to reproduce the measurement curves. The non-linearity for the exchange rate between hepatocytes and bile canaliculi cannot be replaced with a constant exchange rate. As shown in Figure 16 in the Supplementary material, neither zero-order nor first-order kinetics would be valid. In the control group, the excretion and excretion rate vary during the entire simulation time following the concentration curve. In the bile duct ligation group, the excretion reaches a constant value immediately i.e the saturated regime is rapidly reached. The non-linearity enables to reproduce different behaviors of ICG dynamics in the liver.

420

Sensitivity analysis. Generalized sensitivity functions have indicated correlation between the three parameters affecting the bile canaliculi compartment, $Q_{h b}, \mathrm{~S}$ and $F_{b c}$. Overall, the strongest correlation is between $Q_{h b}$ and $S$, while the exchange rate $K_{s h}$ between sinusoids and hepatocytes seems uncorrelated from the others. Usually the excretion rate $Q_{h b} /\left(1+S C_{h}\right)$ is expressed as $V_{\max } / K_{m}+C_{h}$. Our choice was motivated by the sensitivity analysis that showed that the correlation was reduced (but still present) with the chosen formulation. The inverse problems on synthetic curves tend to confirm the sensitivity analysis. Indeed, in the first case, when all parameters are estimated, $F_{b c}$ and $K_{s h}$ are correctly estimated. Whereas $\mathrm{S}$ and $Q_{h b}$ estimates are unsatisfactory, confirming that the correlation between these two parameters make their estimation difficult. The next inverse problems have revealed that, adding information about what happens inside or goes out of the liver overall compartment, i.e. knowing $K_{s h}$ or $F_{b c}$ improves the estimation of the other 
parameters. In the first case, when $K_{s h}$ is known, the saturation parameter $\mathrm{S}$ seems harder to estimate than the other parameters. Moreover, the confidence in its estimation is small (Figure 12 E Appendix).

${ }_{435}$ In the second case, which corresponds to a regime that rapidly transitions to zero-order kinetics, i.e. in which the term $S C_{h}$ becomes rapidly larger than 1, when all parameters are estimated only the sinusoid-hepatocyte exchange rate $K_{s h}$ is correctly estimated, which is coherent with the sensitivity functions (Figure 4). When the bile canaliculi outflow $F_{b c}$ is known in the second case, $K_{s h}$ is still correctly estimated and $Q_{h b}$ and S are overestimated. However, the ratio of these two parameters, ${ }_{440} \frac{Q_{h b}}{S}$, is well estimated. In addition, the observation curve is correctly fitted. This result confirms the correlation between $Q_{h b}$ and $S$, even if $F_{b c}$ is known. In this regime, the observation is more sensitive to the parameters ratio than to the parameters. Indeed, as rapidly during the simulation $S C_{h}$ becomes greater than 1 , it leads to $\frac{Q_{h b}}{1+S C_{h}} C_{h} \simeq Q_{h b} / S$. In addition, due to the added noise (and correlations), more than one set of parameters can fit correctly the noisy observation curve. One

${ }_{445}$ would need more precise data during the transition regime to estimate the parameters separately. To conclude, the inverse problems on noisy synthetic data have verified the method and confirmed the sensitivity analysis conclusions: with the liver ICG amount as observation, it is difficult - maybe ill-posed - to identify the four parameters. However, knowing $F_{b c}$ or $K_{s h}$ parameters, leads to an estimation of the other parameters, with three runs of UKF algorithm, and the observation curve is well fitted.

Data measurements and model validation. Once the approach has been verified on synthetic data, the in-vivo measurements from (El-Desoky et al. (1999)) have been used as observations. Since the inverse problems and the sensitivity analysis have revealed that the four parameters are unlikely to 455 be all estimated, two individual curves, each from different pathophysiological groups, are used to identify the unknown parameters of the mathematical model. The bile duct ligation group curve seems similar to the second regime type of curve. In that case, according to the inverse problem on synthetic observations, the observation curve is well fitted when the bile canaliculi flow $F_{b c}$ parameter is known. Moreover, $F_{b c}$ is known for this group since the bile duct has been ligated. The control group curve is similar to the first regime curve. According to the inverse problems on synthetic observations to fix $K_{s h}$ improves the parameter estimation. Therefore, these parameters are fixed respectively for each group, and 100 estimation procedures have been performed to identify parameter ranges for each group. The Michaelis Menten parameters are compared between the 
control and bile duct ligation groups. Both $V_{\max }$ and $K_{m}$ are much smaller in the bile duct ligation group compared with the control group estimations. Due to a reduction in $K_{m}$, saturation (meaning when the reaction rate is proportionally close to $\left.V_{\max }\right)$ is reached for a smaller concentration in the bile duct ligation group than in the control group. Indeed, in Supplementary Figure 16, the exchange term peaks at $100 \mathrm{Au} \mathrm{ml} / \mathrm{s}$ for the control group, quite far from the $V_{\max }=Q_{h b} / S \sim 610 \mathrm{Au} \mathrm{ml} / \mathrm{s}$; whereas for the bile ligation group, the exchange term is rapidly around $0.107 \mathrm{Au} \mathrm{ml} / \mathrm{s}$, exactly the value of $V_{\max }=Q_{h b} / S$, despite the fact that during that time period (less than 1 minute), the hepatic cell concentration is similar in both groups (Figure 8). According to the simulation results, when saturation is reached, the excretion rate to bile canaliculi is reduced, in the case of bile duct ligation (Supplementary Figure 16). ICG specific carriers are for example MDRP2 and MDR3 (De Gasperi et al. (2016)). Inhibition from other compounds, which share the same carrier as ICG, might explain the decrease of $V_{\max }$ and $K_{m}$ parameters that are linked to a change in activity of enzyme and/or transporters for ICG (De Gasperi et al. (2016)). Indeed, the export pumps for ICG are also required for bilirubin excretion among others (Boyer (2013). Therefore the excretion rate is reduced, with the reduction of $K_{m}$ and $V_{\max }$ (meaning saturation is reached for smaller concentration and less ATP is available). In this work, $K_{s h}$ is the same in both groups, but this assumption should be confirmed by further analysis, based on experiments for example like proposed by (Reif et al. (2016)). Besides, only one experimental curve per group was published in (El-Desoky et al. (1999)). Hence, the parameter variance (inter-subject variability) could not be estimated within each group.

Thanks to the model, the impact of bile duct ligation on the different liver compartments can be assessed (with the assumption that $K_{s h}$ is the same for both groups). Figure 8 shows the ICG amount over time in the sinusoids, the hepatocytes and the bile canaliculi compartments for the control and the bile duct ligation groups. In the control group simulation (solid lines), the bile canaliculi compartment contains less ICG than in the bile duct ligation group (dash lines) after 8 minutes. In the control group the amount of ICG is higher in the hepatocytes than in the bile canaliculi or in the sinusoids compartment. A second observation is that in the control group, the ICG quantity decreases over time in all compartments. Whereas, in the bile duct ligation group, the ICG is stored in the hepatocytes, and as a consequence also remains in high amount in the sinusoids (because of the balance between sinusoids and hepatocytes concentration). This leads to a large amount of ICG in the rest of the blood circulation. 

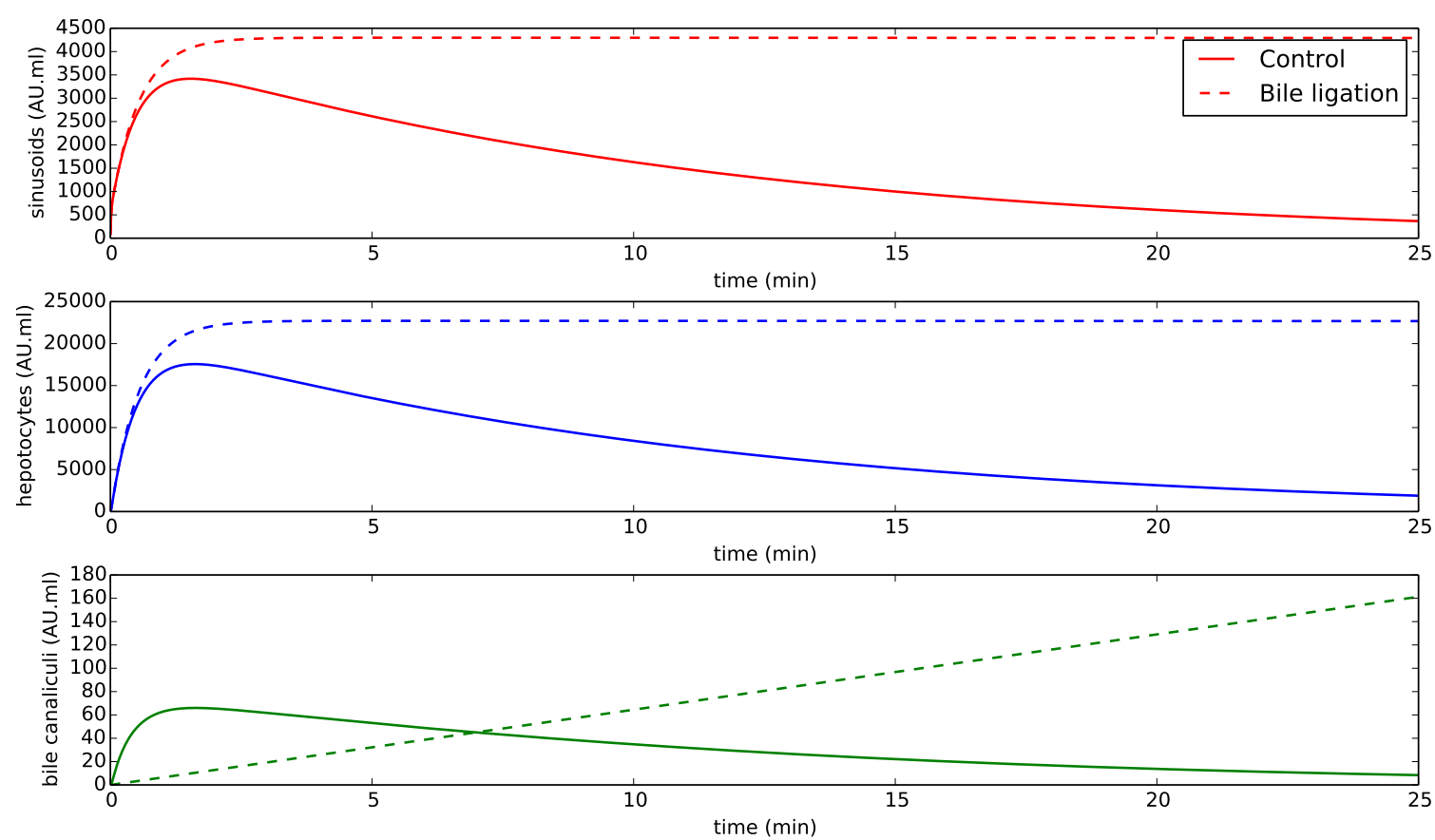

Figure 8: Model simulations of ICG amount over time in the different compartments of the liver. ICG amount over time of sinusoids, hepatocytes and bile canaliculi for the control simulation (solid lines) and the bile duct ligation simulation (dash lines). 
The small decay in the bile duct ligation group measurement curve is not reproduced with the model, by construction (Figure 5 left). This small decrease can be due to fluorescence light intensity decay over time. Indeed, if the common bile duct is completely ligated, no ICG can exit the liver within the bile, thus no decrease should appear in the curve. In the model, because the bile canaliculi Desoky et al. (1999)). However, the large standard deviation in the observation within rabbits of the group with portal vein partial occlusion, makes the analysis difficult.

ICG and liver function evaluation. A common test to evaluate liver function is the percentage of group, the differences appear after a few minutes. The plasma disappearance curve of ICG after a 


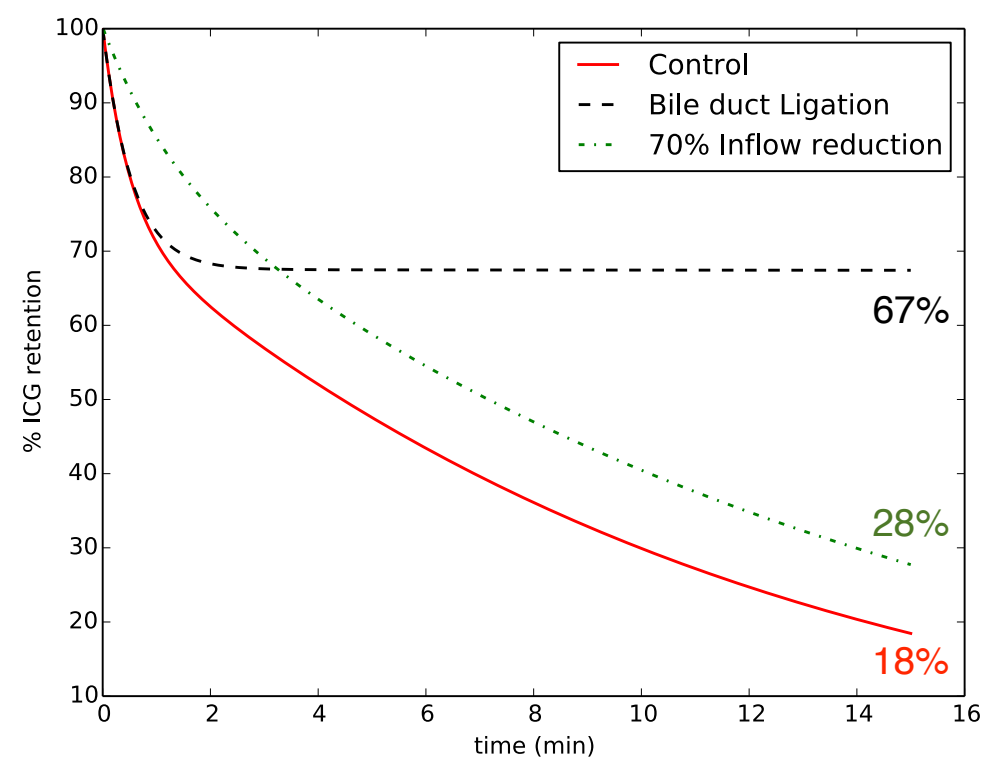

Figure 9: Model simulations of \% of ICG remaining in blood over $15 \mathrm{~min}$. The simulation is performed for different groups: control group (solid red), bile duct ligation group (dash line black) and $70 \%$ inflow reduction group (dash line green).

single intravenous bolus reported by (Kawasaki et al. (1984); Imamura et al. (2005)) has the same behavior. ICG retention is $50 \%$ higher in the bile duct ligation group than in the inflow reduction group. In addition, in both of these groups, the value is larger than in the control group.

According to these results, if the unhealthy liver problem is related to bile secretion, for the first two minutes the ICG retention should be similar to the "normal" value and then stagnate. Whereas, if the problem is related to liver perfusion (porto-caval shunts, portal thrombosis, hepatic arterial stenosis, etc), already after two minutes the percentage of ICG retention should be higher than for a healthy liver.

Extrapolation to clinical applications. In this work, model parameters were estimated from in-vivo ICG fluorescence measurements on rabbit liver. The measurement of liver fluorescence after intravenous injection of ICG can be applied to the clinics, as proposed by (Kawaguchi et al. (2013)). In our parameter estimation procedure one of the parameters, $K_{s h}$ or $F_{b c}$, was known. Indeed, according to the sensitivity analysis, with the observation of the fluorescence in the liver tissue not all parameters can be estimated. Therefore, to apply the method described above to clinical cases more information is required. However, in patients with bile duct obstruction, the proposed method could 
be applied since the bile canaliculi flow $\left(F_{b c}\right)$ is known. Nevertheless we think that performing invitro or similar study to improve knowledge on ICG transport and excretion processes could enable to determine some parameters of the model. We are currently working on measurement protocols to improve parameter estimations, together with liver surgeons (Bekheit and Vibert (2015)). For now, real-time imaging of ICG fluorescence has been used during liver surgery for a number applications (Bekheit and Vibert (2015)), e.g. to identify liver tissue zones with perfusion issues (Kawaguchi et al. (2013)), to detect small superficial liver nodules and to distinguish malignant from benign lesions (Lim et al. (2014)), to evaluate the liver condition at reperfusion during transplantation, etc. To our knowledge, the quantification of hepatic function with ICG fluorescence has not been performed yet in the clinics. Combining this technique with a mathematical model and parameter estimation procedure might provide a novel estimation of the liver function(s) peri-operatively. Moreover, it could constitute an additional tool to quantify which function of the liver is affected and by how much in drug-induced toxicity studies: animal studies have shown that drug-induced cholestasis can come from sinusoid-hepatocyte or hepatocyte-bile uptake and effluxes (Jansen and Sturm (2003)).

To conclude, the sensitivity analysis combined with solving the inverse problem on synthetic data, provides a good understanding of model parameters dependency. The proposed three-compartment model for the liver is able to reproduce the different types of measurements from (El-Desoky et al. (1999)). According to the model and the parameter estimation procedure, saturation is reached for smaller concentrations in the bile duct ligation group than in the control group. This leads to a reduced excretion coefficient from hepatocytes to bile canaliculi. The reduction of excretion of ICG might be linked the competition of ICG with other compounds. To our knowledge it is the first time that a sequential approach is chosen to solve an inverse problem based on ICG liver amount over time, that leads to an estimation of pharmacokinetics parameter ranges for ICG. According to the numerical simulations, the liver inflow issue is likely to induce an initial deviation of ICG percentage retention dynamics whereas a bile secretion issue is likely to cause a slowing down of the ICG percentage retention decrease after a few minutes. Finally, mathematical modeling and numerical simulation of ICG processing by the liver, are promising tools to improve knowledge on the relation between ICG processing and hepatic "functions".

\section{Acknowledgment}

The authors gratefully acknowledge Dr. Sanjay Pant for his expertise in Kalman filter algorithm and sensitivity analysis as well as for his implementation of UKF in Python. 
Funding: This work was supported by the National Grant ANR-13-TECS-0006 (iFlow).

\section{References}

Alacam, B., Yazici, B., Intes, X., Chance, B., 2006. Extended kalman filtering for the modeling and analysis of icg pharmacokinetics in cancerous tumors using nir optical methods. IEEE transactions on biomedical engineering 53, 1861-1871.

Alander, J.T., Kaartinen, I., Laakso, A., Pätilä, T., Spillmann, T., Tuchin, V.V., Venermo, M., Välisuo, P., 2012. A review of indocyanine green fluorescent imaging in surgery. Int J Biomed Imaging 2012, 940585. doi $10.1155 / 2012 / 940585$

Bai, P., Banks, H.T., Dediu, S., Govan, A.Y., Last, M., Lloyd, A.L., Nguyen, H.K., Olufsen, M.S., Rempala, G., Slenning, B.D., 2007. Stochastic and deterministic models for agricultural production networks. Math Biosci Eng 4, 373-402.

Bekheit, M., Vibert, E., 2015. Fluorescent-guided liver surgery: Paul brousse experiences and perspective, in: Fluorescence Imaging for Surgeons. Springer, pp. 117-126.

Bertoglio, C., Moireau, P., Gerbeau, J.F., 2012. Sequential parameter estimation for fluid-structure problems: Application to hemodynamics. International Journal for Numerical Methods in Biomedical Engineering 28, 434-455.

Bezy-Wendling, J., Kretowski, M., Mescam, M., Jurczuk, K., Eliat, P.A., 2007. Simulation of hepatocellular carcinoma in mri by combined macrovascular and pharmacokinetic models, in: Biomedical Imaging: From Nano to Macro, 2007. ISBI 2007. 4th IEEE International Symposium on, IEEE. pp. 1272-1275.

Burns, E., Triger, D., Tucker, G., Bax, N., 1991. Indocyanine green elimination in patients with liver disease and in normal subjects. Clinical Science 80, 155-160.

Cooke, A.R., Harrison, D.D., Skyring, A.P., 1963. Use of indocyanine green as a test of liver function. Am J Dig Dis 8, 244-50.

De Gasperi, A., Mazza, E., Prosperi, M., 2016. Indocyanine green kinetics to assess liver function: Ready for a clinical dynamic assessment in major liver surgery? World journal of hepatology 8 , 355. 
Diehl, K.H., Hull, R., Morton, D., Pfister, R., Rabemampianina, Y., Smith, D., Vidal, J.M., van de Vorstenbosch, C., European Federation of Pharmaceutical Industries Association and European Centre for the Validation of Alternative Methods, 2001. A good practice guide to the administration of substances and removal of blood, including routes and volumes. J Appl Toxicol 21, 15-23.

El-Desoky, A., Seifalian, A.M., Cope, M., Delpy, D.T., Davidson, B.R., 1999. Experimental study of liver dysfunction evaluated by direct indocyanine green clearance using near infrared spectroscopy. Br J Surg 86, 1005-11. doi:10.1046/j.1365-2168.1999.01186.x.

Ghallab, A., Cellière, G., Henkel, S.G., Driesch, D., Hoehme, S., Hofmann, U., Zellmer, S., Godoy, P., Sachinidis, A., Blaszkewicz, M., Reif, R., Marchan, R., Kuepfer, L., Häussinger, D., Drasdo, D., Gebhardt, R., Hengstler, J.G., 2016. Model-guided identification of a therapeutic strategy to reduce hyperammonemia in liver diseases. J Hepatol 64, 860-71. doi:10.1016/j.jhep.2015.11. 018

Grainger, S., Keeling, P., Brown, I., Marigold, J., Thompson, R., 1983. Clearance and non-invasive determination of the hepatic extraction of indocyanine green in baboons and man. Clinical science (London, England: 1979) 64, 207-212.

Hammad, S., Hoehme, S., Friebel, A., Von Recklinghausen, I., Othman, A., Begher-Tibbe, B., Reif, R., Godoy, P., Johann, T., Vartak, A., et al., 2014. Protocols for staining of bile canalicular and sinusoidal networks of human, mouse and pig livers, three-dimensional reconstruction and quantification of tissue microarchitecture by image processing and analysis. Archives of toxicology $88,1161-1183$.

Haykin, S.S., et al., 2001. Kalman filtering and neural networks. Wiley Online Library.

Hindmarsh, A.C., Brown, P.N., Grant, K.E., Lee, S.L., Serban, R., Shumaker, D.E., Woodward, C.S., 2005. SUNDIALS: Suite of nonlinear and differential/algebraic equation solvers. ACM Transactions on Mathematical Software (TOMS) 31, 363-396.

Hoekstra, L.T., de Graaf, W., Nibourg, G.A.A., Heger, M., Bennink, R.J., Stieger, B., van Gulik, T.M., 2013. Physiological and biochemical basis of clinical liver function tests: a review. Ann Surg 257, 27-36. doi:10.1097/SLA.0b013e31825d5d47. 
Imamura, H., Sano, K., Sugawara, Y., Kokudo, N., Makuuchi, M., 2005. Assessment of hepatic reserve for indication of hepatic resection: decision tree incorporating indocyanine green test. Journal of Hepato-Biliary-Pancreatic Sciences 12, 16-22.

Jansen, P.L., Sturm, E., 2003. Genetic cholestasis, causes and consequences for hepatobiliary transport. Liver international 23, 315-322.

Julier, S., Uhlmann, J., Durrant-Whyte, H.F., 2000. Technical notes and correspondence : A new method for the nonlinear transformation of means and covariances in filters and estimators. IEEE Transactions on automatic control 45, 477 .

Julier, S.J., Uhlmann, J.K., Durrant-Whyte, H.F., 1995. A new approach for filtering nonlinear systems, in: American Control Conference, Proceedings of the 1995, IEEE. pp. 1628-1632.

Kawaguchi, Y., Ishizawa, T., Miyata, Y., Yamashita, S., Masuda, K., Satou, S., Tamura, S., Kaneko, J., Sakamoto, Y., Aoki, T., et al., 2013. Portal uptake function in veno-occlusive regions evaluated by real-time fluorescent imaging using indocyanine green. Journal of hepatology 58, 247-253.

Kawasaki, S., Umekita, N., Beppu, T., Wada, T., Sugiyama, Y., Iga, T., Hanano, M., 1984. Hepatic transport of indocyanine green in dogs chronically intoxicated with dimethylnitrosamine. Toxicology and applied pharmacology 75, 309-317.

Lim, C., Vibert, E., Azoulay, D., Salloum, C., Ishizawa, T., Yoshioka, R., Mise, Y., Sakamoto, Y., Aoki, T., Sugawara, Y., et al., 2014. Indocyanine green fluorescence imaging in the surgical management of liver cancers: current facts and future implications. Journal of visceral surgery $151,117-124$.

Miao, H., Xia, X., Perelson, A.S., Wu, H., 2011. On identifiability of nonlinear ode models and applications in viral dynamics. SIAM Rev Soc Ind Appl Math 53, 3-39. doi 10.1137/090757009.

Moireau, P., Chapelle, D., 2011. Reduced-order unscented kalman filtering with application to parameter identification in large-dimensional systems. ESAIM: Control, Optimisation and Calculus of Variations 17, 380-405.

Moody, F.G., Rikkers, L.F., Aldrete, J.S., 1974. Estimation of the functional reserve of human liver. Ann Surg 180, 592-8. 
Ott, P., Keiding, S., Johnsen, A.H., Bass, L., 1994. Hepatic removal of two fractions of indocyanine green after bolus injection in anesthetized pigs. Am J Physiol 266, G1108-22.

Pant, S., Corsini, C., Baker, C., Hsia, T.Y., Pennati, G., Vignon-Clementel, I.E., 2017. Inverse problems in reduced order models of cardiovascular haemodynamics: aspects of data assimilation and heart rate variability. Journal of The Royal Society Interface 14, 20160513.

Pant, S., Fabrèges, B., Gerbeau, J.F., Vignon-Clementel, I.E., 2014. A methodological paradigm for patient-specific multi-scale cfd simulations: from clinical measurements to parameter estimates for individual analysis. Int J Numer Method Biomed Eng 30, 1614-48. doi 10.1002/cnm.2692.

Reif, R., Ghallab, A., Beattie, L., Günther, G., Kuepfer, L., Kaye, P.M., Hengstler, J.G., 2016. In vivo imaging of systemic transport and elimination of xenobiotics and endogenous molecules in mice. Archives of Toxicology, 1-18.

Sacerdoti, D., Serianni, G., Gaiani, S., Bolognesi, M., Bombonato, G., Gatta, A., 2007. Thrombosis of the portal venous system. Journal of Ultrasound 10, 12-21.

Schwen, L.O., Krauss, M., Niederalt, C., Gremse, F., Kiessling, F., Schenk, A., Preusser, T., Kuepfer, L., 2014. Spatio-temporal simulation of first pass drug perfusion in the liver. PLoS Comput Biol 10, e1003499.

Schwen, L.O., Schenk, A., Kreutz, C., Timmer, J., Rodríguez, M.M.B., Kuepfer, L., Preusser, T., 2015. Representative sinusoids for hepatic four-scale pharmacokinetics simulations. PloS one 10, $\mathrm{e} 0133653$.

675 Shinohara, H., Tanaka, A., Kitai, T., Yanabu, N., Inomoto, T., Satoh, S., Hatano, E., Yamaoka, Y., Hirao, K., 1996. Direct measurement of hepatic indocyanine green clearance with near-infrared a spectroscopy: separate evaluation of uptake and removal. Hepatology 23, 137-44. doi 10.1053/ jhep.1996.v23.pm0008550033.

Simon, D., 2010. Kalman filtering with state constraints: a survey of linear and nonlinear algorithms. IET Control Theory and Applications 4, 1303-1318.

Thomaseth, K., Cobelli, C., 1999. Generalized sensitivity functions in physiological system identification. Annals of biomedical engineering 27, 607-616. 
Tucker, O., Heaton, N., 2005. The 'small for size'liver syndrome. Current opinion in critical care $11,150-155$.

685 Webster, S.H., Liljegren, E.J., Zimmer, D.J., 1947. Organ; body weight ratios for liver, kidneys and spleen of laboratory animals; albino rat. Am J Anat 81, 477-513.

Weiss, M., Krejcie, T.C., Avram, M.J., 2011. A physiologically based model of hepatic icg clearance: Interplay between sinusoidal uptake and biliary excretion. European Journal of Pharmaceutical Sciences 44, 359-365.

\section{Appendix}

For each parameter estimation, the displayed results are: the model observable evolution after the UKF correction step (i.e. while the parameters are being estimated) (plot A), the solution of the mathematical model (direct problem) with the final parameter estimates (plot B), and the evolution in time of the geometric mean $+/$ - geometric standard deviation of the 4 estimated parameters (plots C, D, E, F). The observation curve is also plotted as well as the parameter values from the synthetic observation (known values). The parameters are correctly estimated if the estimated value converges after some time to the known value of the parameter (represented in the following figures with dash line). In addition the confidence in the estimated parameter is increased when the variance is reduced (represented in the following figures by the grey zones). 

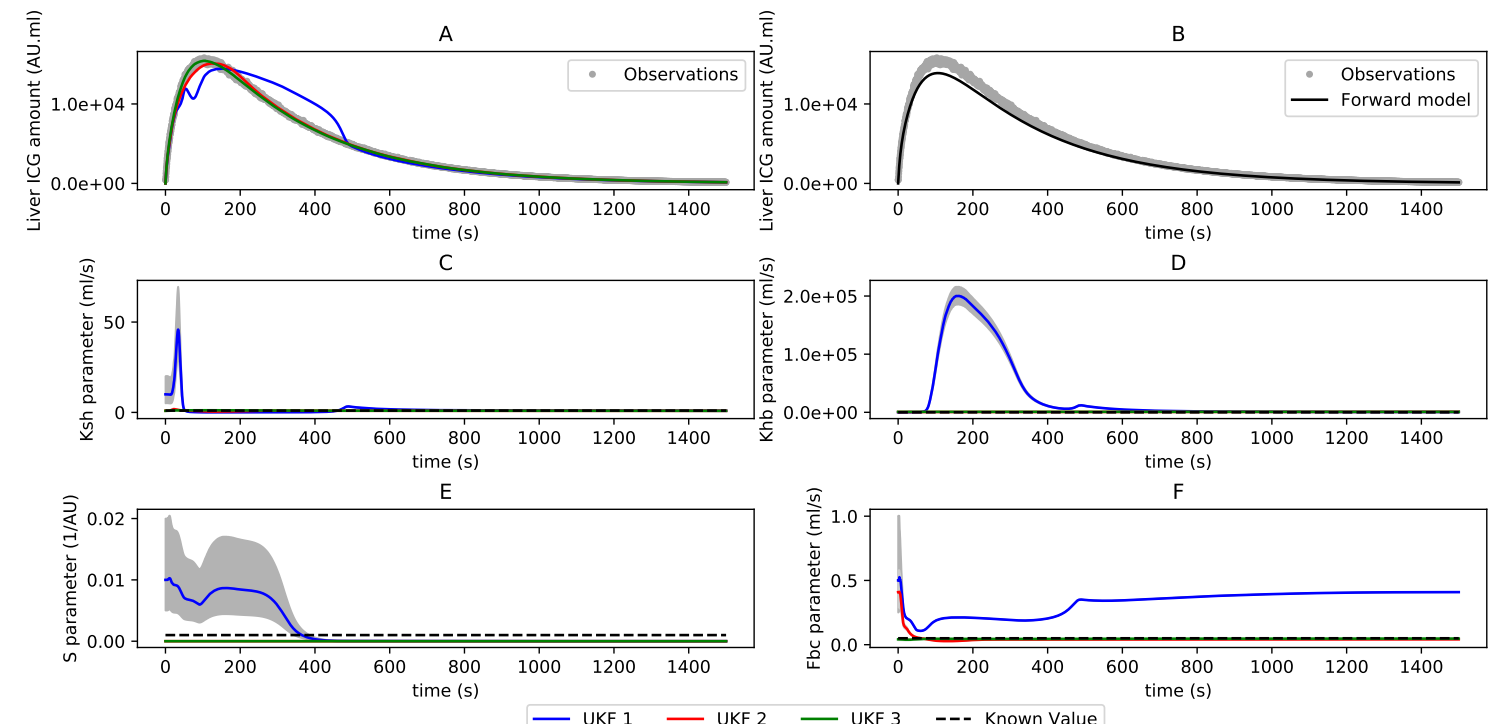

Figure 10: Case 1 with all parameters being estimated. A: ICG amount in the liver over time, observation curve (grey) and results from UKF runs (solid line, first UKF run in blue, second UKF run in red and third UKF run in green); B: ICG amount in the liver over time, observation curve (grey) and direct model solution with parameters from the last UKF run estimation (black solid line). Evolution over time of geometric mean (solid line, first UKF run in blue, second UKF run in red and third UKF run in green) and +/- geometric standard deviation (grey zone) of the estimated parameters for: $K_{s h}(\mathrm{C}) ; Q_{h b}(\mathrm{D}) ; S(\mathrm{E})$ and $F_{b c}(\mathrm{~F})$. Parameter known values (synthetic data parameter set) are plotted with dotted lines. 

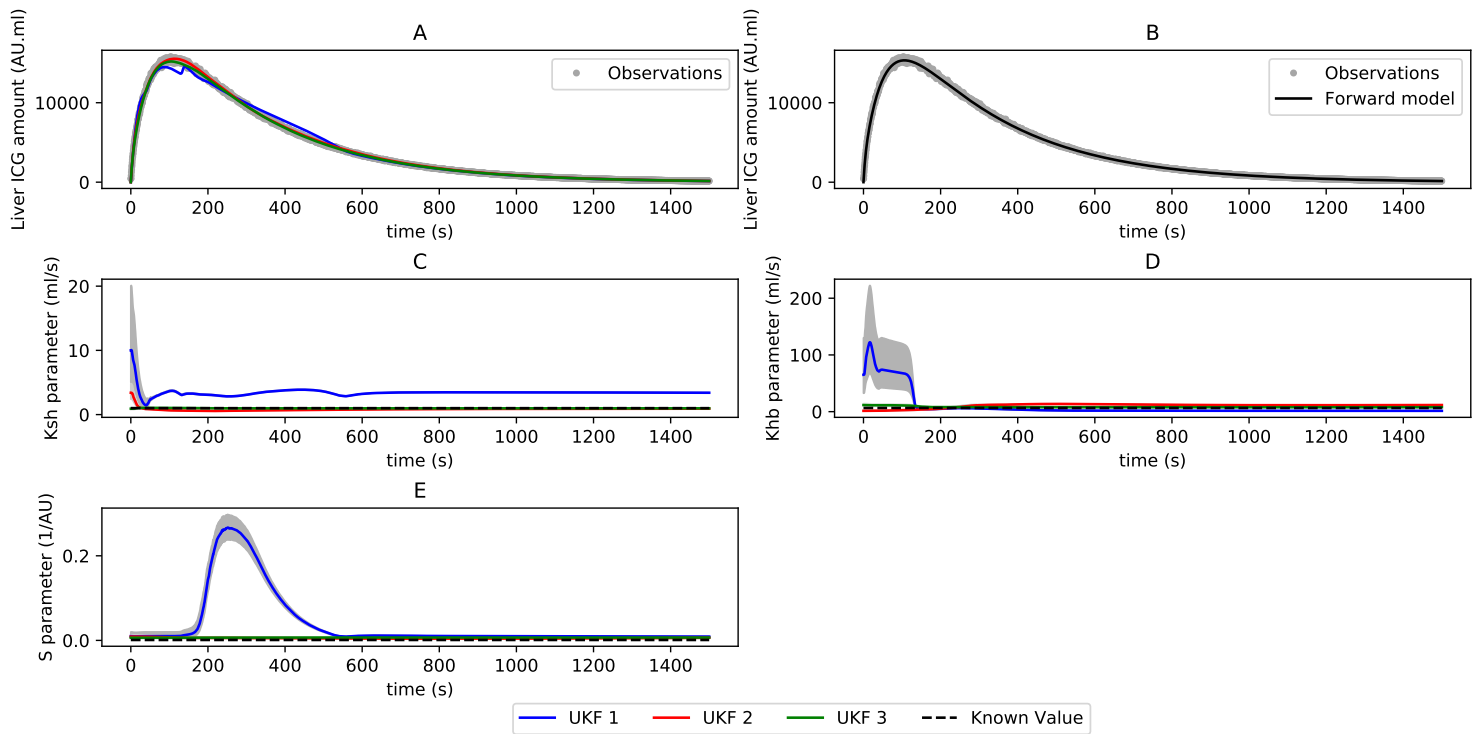

Figure 11: Case 1 with the $F_{b c}$ parameter being known. A: ICG amount in the liver over time, observation curve (grey) and results from UKF runs (solid line, first UKF run in blue, second UKF run in red and third UKF run in green); B: ICG amount in the liver over time, observation curve (grey) and direct model solution with parameters from the last UKF run estimation (black solid line). Evolution over time of geometric mean (solid line, first UKF run in blue, second UKF run in red and third UKF run in green) and +/- geometric standard deviation (grey zone) of the estimated parameters for: $K_{s h}(\mathrm{C}) ; Q_{h b}(\mathrm{D})$ and $S(\mathrm{E})$. Parameters known values (synthetic data parameter set) are plotted with dotted lines. 

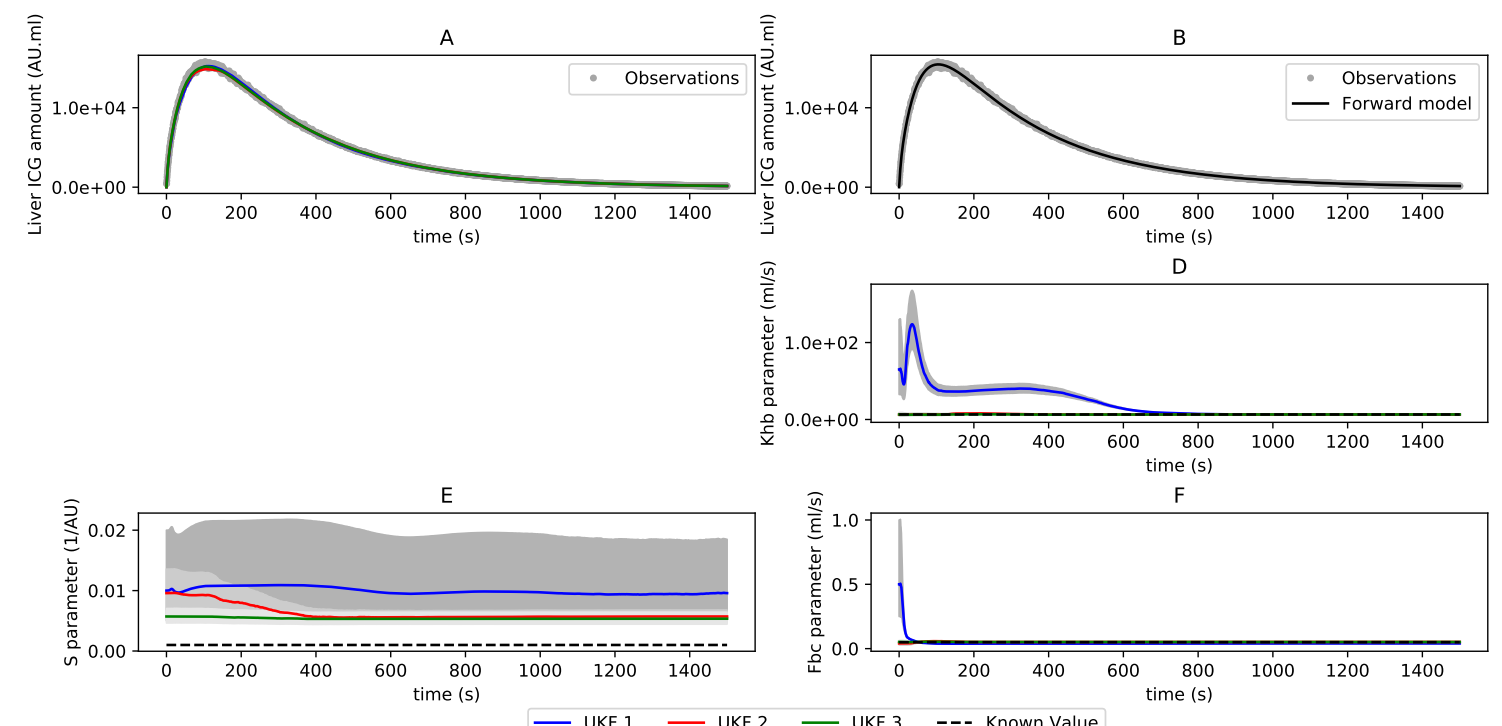

Figure 12: Case 1 with the $K_{s h}$ parameter being known. A: ICG amount in the liver over time, observation curve (grey) and results from UKF runs (solid line, first UKF run in blue, second UKF run in red and third UKF run in green); B: ICG amount in the liver over time, observation curve (grey) and direct model solution with parameters from last UKF run estimation (black solid line). Evolution over time of geometric mean (solid line, first UKF run in blue, second UKF run in red and third UKF run in green) and +/- geometric standard deviation (grey zone) of the estimated parameters for: $Q_{h b}(\mathrm{D}) ; S(\mathrm{E})$ and $F_{b c}(\mathrm{~F})$. Parameters known values (synthetic data parameter set) are plotted with dotted lines. 

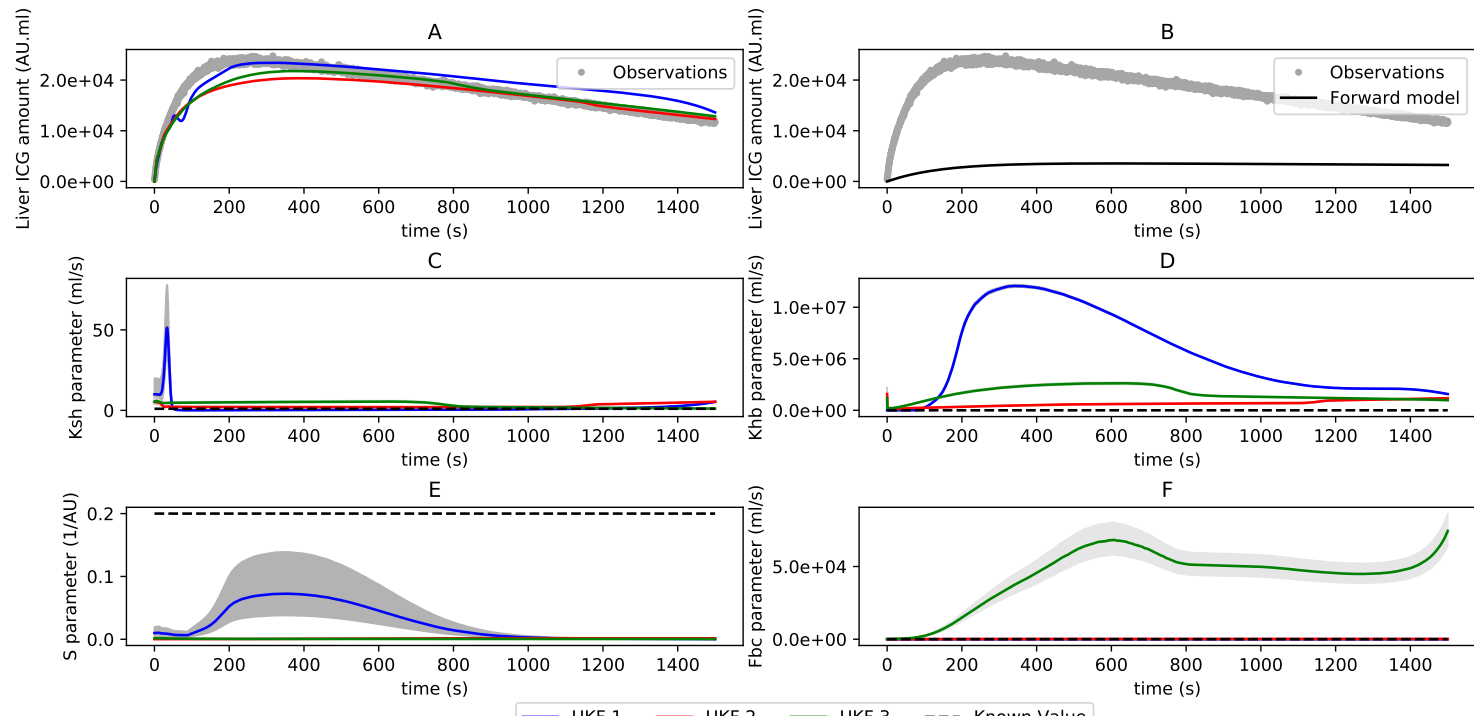

Figure 13: Case 2 with all parameters being estimated. A: ICG amount in the liver over time, observations curve (grey) and results from UKF runs (solid line, first UKF run in blue, second UKF run in red and third UKF run in green); B: ICG amount in the liver over time, observations curve (grey) and direct model solution with parameters from last UKF run estimation (black solid line). Evolution over time of geometric mean (solid line, first UKF run in blue, second UKF run in red and third UKF run in green) and +/- geometric standard deviation (grey zone) of the estimated parameters for: $K_{s h}(\mathrm{C}) ; Q_{h b}(\mathrm{D}) ; S(\mathrm{E})$ and $F_{b}(\mathrm{~F})$. Parameters known values (synthetic data parameter set) are plotted with dotted lines. 

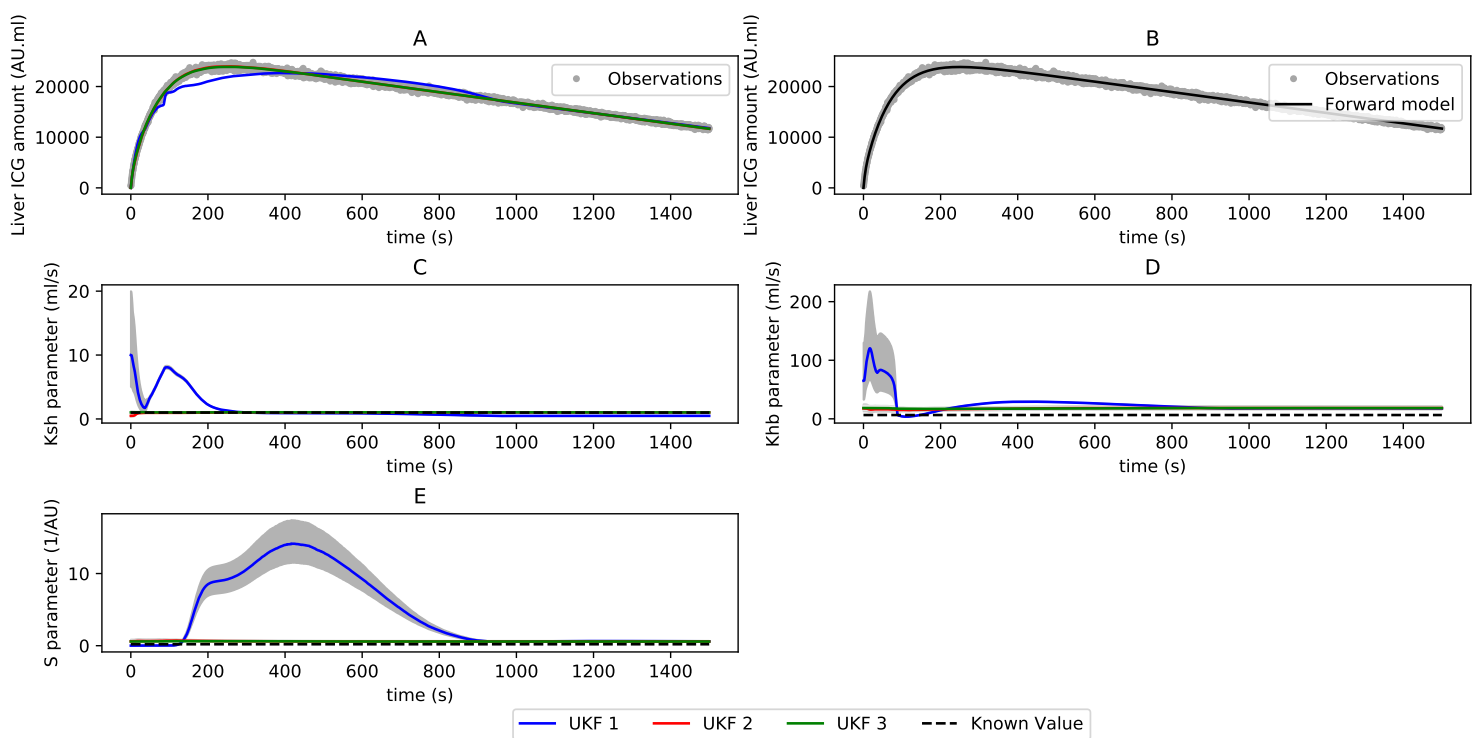

Figure 14: Case 2: $F_{b c}$ parameter is known. A: ICG amount in the liver over time, observation curve (grey) and results from UKF runs (solid line, first UKF run in blue, second UKF run in red and third UKF run in green); B: ICG amount in the liver over time, observation curve (grey) and direct model solution with parameters from last UKF run estimation (black solid line). Evolution over time of geometric mean (solid line, first UKF run in blue, second UKF run in red and third UKF run in green) and +/- geometric standard deviation (grey zone) of the estimated parameters for: $K_{s h}(\mathrm{C}) ; Q_{h b}(\mathrm{D})$ and $S(\mathrm{E})$. Parameters known values (synthetic data parameter set) are plotted with dotted lines. 

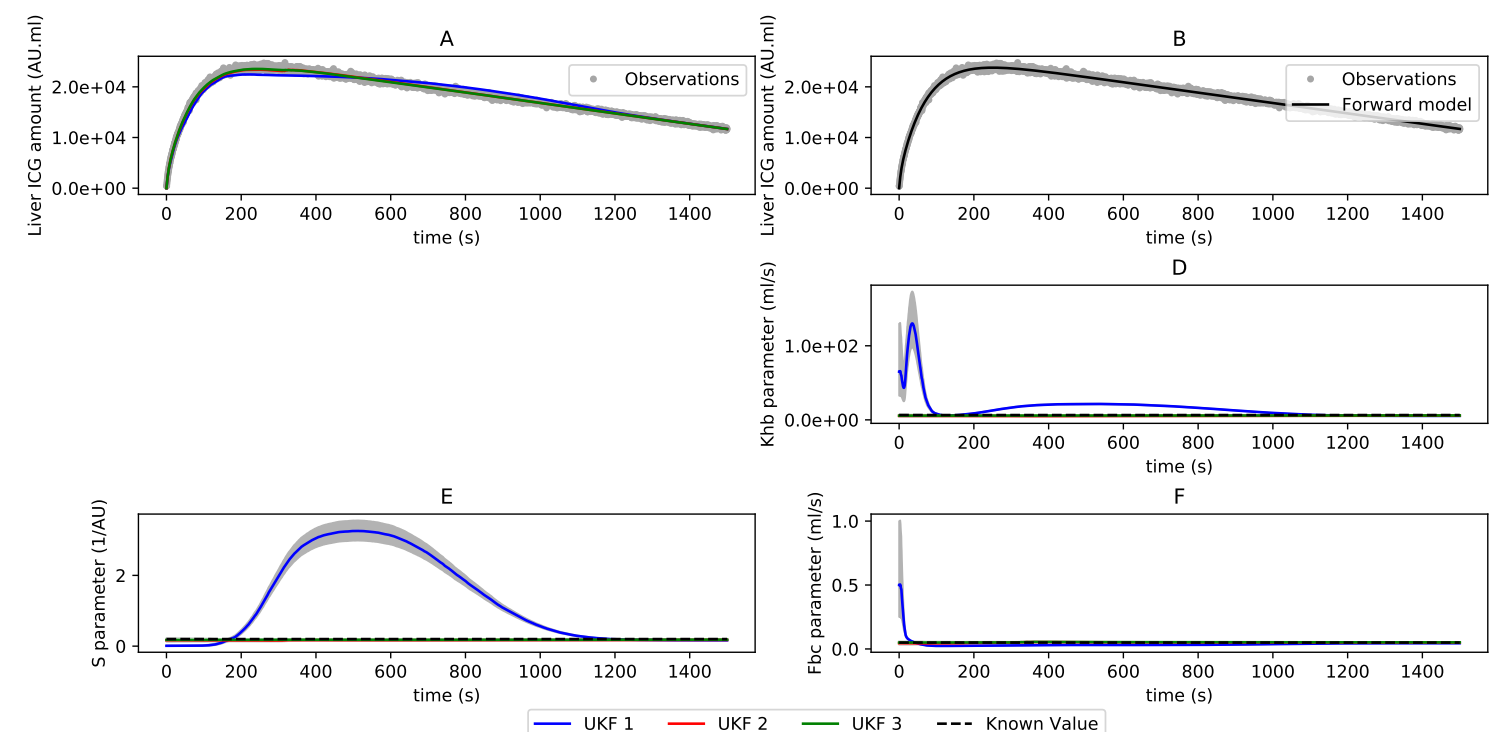

Figure 15: Case 2 with $K_{s h}$ parameter being known. A: ICG amount in the liver over time, observation curve (grey) and results from UKF runs (solid line, first UKF run in blue, second UKF run in red and third UKF run in green); B: ICG amount in the liver over time, observation curve (grey) and direct model solution with parameters from last UKF run estimation (black solid line). Evolution over time of geometric mean (solid line, first UKF run in blue, second UKF run in red and third UKF run in green) and +/- geometric standard deviation (grey zone) of the estimated parameters for: $Q_{h b}(\mathrm{D}) ; S(\mathrm{E})$ and $F_{b c}(\mathrm{~F})$. Parameters known values (synthetic data parameter set) are plotted with dotted lines. 
The ICG mathematical model proposed in this work and the sensitivity analysis tools are available in open-access in the github repository https://github.com/caudebert/LiverICG-CompartmentModel.

The figure 16 gives information on the type of kinetics for the ICG transfer between hepatocytes and bile canaliculi. As commented in the main text, both the exchange and exchange rate vary over time in the control group. The behavior is closest to first-order kinetics (exchange rate does not vary much). By contrast, in bile duct ligation, saturation (zero-order kinetics) occurs very rapidly. Hence, to capture all behaviors, a nonlinear model is needed.
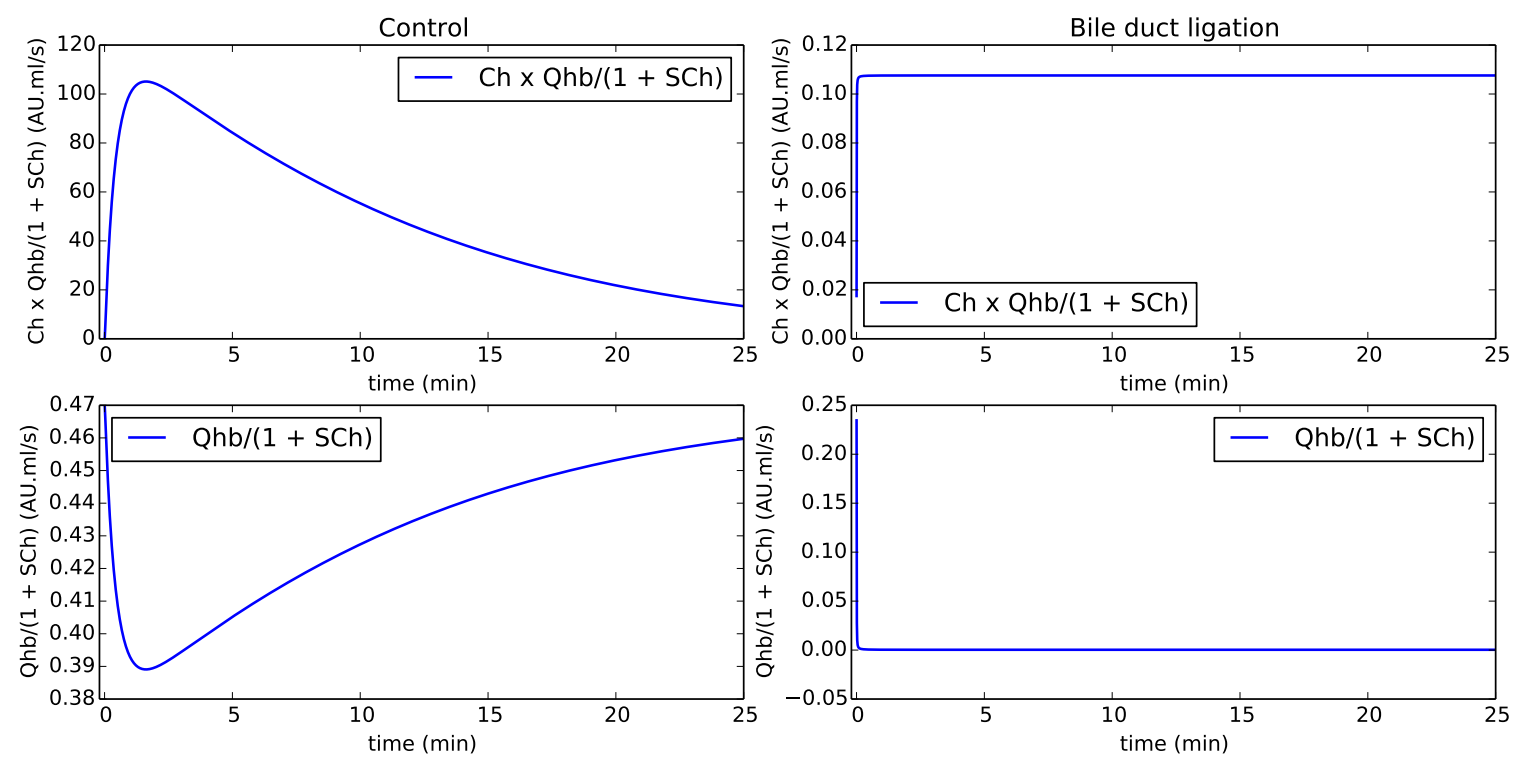

Figure 16: Hepatocytes - bile canaliculi exchange coefficient over time. The exchange term $\frac{C_{h}(t) Q_{h b}}{1+S C_{h}(t)}$ and exchange rate $\frac{Q_{h b}}{1+S C_{h}(t)}$ over time for the control and bile duct ligation groups. 